\title{
Revisiting the Relationship Between Correlation Coefficient, Confidence Level, and Sample Size
}

Qifan Yang, ${ }^{1,2}$ Minyi Su, ${ }^{1,2}$ Yan $\mathrm{Li}^{1,3}$ Renxiao Wang ${ }^{1,3,4 *}$

${ }^{1}$ State Key Laboratory of Bioorganic and Natural Products Chemistry, Center for Excellence in Molecular Synthesis, Shanghai Institute of Organic Chemistry, Chinese Academy of Sciences, 345 Lingling Road, Shanghai 200032, People's Republic of China;

${ }^{2}$ University of Chinese Academy of Sciences, Beijing 100049, People's Republic of China;

3 Department of Medicinal Chemistry, School of Pharmacy, Fudan University, 826 Zhangheng Road, Shanghai 201203, People's Republic of China

${ }^{4}$ Shanxi Key Laboratory of Innovative Drugs for the Treatment of Serious Diseases Basing on Chronic Inflammation, College of Traditional Chinese Medicines, Shanxi University of Chinese Medicine, Taiyuan, Shanxi 030619, People's Republic of China

*: Correspondence E-mail: wangrx@mail.sioc.ac.cn; wangrx@fudan.edu.cn

\section{TABLE OF CONTENTS}

PART 1. ESTIMATE OF MINIMUM SAMPLE SIZE BY TWO-SIDED TEST 2

PART 2. POSSIBLE RANGE OF THE INTER-CORRELATION BETWEEN TWO MODELS 4

PART 3. HYPOTHESIS TEST ON MOTHER SETS IN NORMAL DISTRIBUTION 5

PART 4. HYPOTHESIS TEST ON MOTHER SETS IN UNIFORM DISTRIBUTION . .12

PART 5. HYPOTHESIS TEST ON MOTHER SETS IN EXPONENTIAL DISTRIBUTION

PART 6. ANALYSIS OF THE PEARSON CORRELATION COEFFICIENTS OBTAINED ON THE CASF-2013 BENCHMARK 26

PART 7. SOURCE CODES USED IN NUMERICAL SIMULATION 29 


\section{PART 1. ESTIMATE OF MINIMUM SAMPLE SIZE BY TWO-SIDED TEST}

A two-sided test is more reasonable if one wants to the difference between two Pearson correlation coefficients $(R)$ is significant. If the two models under test produce $R_{1}$ and $R_{2}$ on a certain data set, consider the following hypotheses:

$$
H_{0}: \rho_{1}=\rho_{2} \quad \text { and } \quad H_{1}: \rho_{1} \neq \rho_{2}
$$

At a given combination of confidence $(1-\alpha)$ and statistical power $(\beta)$ :

$$
\begin{gathered}
P\left\{|Z|>\mathrm{z}_{\alpha / 2} \mid H_{0} \text { is true }\right\}=\alpha \\
P\left\{Z>\mathrm{z}_{\alpha / 2} \mid H_{1} \text { is true }\right\}=1-\beta \\
\mathrm{z}_{\alpha / 2}=\mu^{*}-\mathrm{z}_{\beta} \text { and thus } \mu^{*}=\mathrm{z}_{\alpha / 2}+\mathrm{z}_{\beta}
\end{gathered}
$$

Then, Dunn \& Clark's test statistic can be computed as:

$$
\mathrm{z}_{\alpha / 2}+\mathrm{z}_{\beta}=\left(F\left(\rho_{2}\right)-F\left(\rho_{1}\right)\right) \times \sqrt{(N-3) / 2(1-c)}
$$

the sample size can be obtained:

$$
N=\frac{2 \times\left(\mathrm{z}_{\alpha / 2}+\mathrm{z}_{\beta}\right)^{2} \times(1-c)}{\left(F\left(\rho_{2}\right)-F\left(\rho_{1}\right)\right)^{2}}+3
$$

Some examples of the minimum sample sizes computed by Eq.S3 are given in Table $\mathrm{S} 1$ and Table $\mathrm{S} 2$ below.

Table S1. Minimum sample sizes required for comparing two models when $\Delta \rho=0.10$ (confidence level $=95 \%$ and power $=0.80$, two-sided test)

\begin{tabular}{ccccccc}
\hline$\rho_{1}$ & 0.8 & 0.7 & 0.6 & 0.5 & 0.4 & 0.3 \\
$\rho_{2}$ & 0.9 & 0.8 & 0.7 & 0.6 & 0.5 & 0.4 \\
$\rho_{12}$ & & & & & & \\
\hline 0.9 & 36 & 63 & 87 & 108 & 125 & 139 \\
0.8 & 63 & 119 & 168 & 210 & 245 & 273 \\
0.7 & 77 & 165 & 241 & 307 & 362 & 406 \\
0.6 & 80 & 202 & 308 & 399 & 475 & 536 \\
0.5 & 72 & 231 & 369 & 487 & 586 & 665 \\
0.4 & & 250 & 423 & 570 & 693 & 791 \\
0.3 & & 261 & 470 & 648 & 797 & 916 \\
0.2 & & 262 & 510 & 722 & 898 & 1039
\end{tabular}




\begin{tabular}{ccccc}
0.1 & 544 & 791 & 996 & 1160 \\
0 & 571 & 855 & 1091 & 1280 \\
-0.1 & 591 & 914 & 1182 & 1397 \\
-0.2 & & 968 & 1271 & 1512 \\
-0.3 & & 1018 & 1356 & 1626 \\
-0.4 & & & 1438 & 1737 \\
-0.5 & & & 1517 & 1847 \\
-0.6 & & & 1955 \\
-0.7 & & & 2061 \\
\hline
\end{tabular}

Table S2. Minimum sample sizes required for comparing two models when $\Delta \rho=0.05$ (confidence level $=95 \%$ and power $=0.80$, two-sided test)

\begin{tabular}{|c|c|c|c|c|c|c|}
\hline$\rho_{1}$ & 0.85 & 0.75 & 0.65 & 0.55 & 0.45 & 0.35 \\
\hline$\rho_{2}$ & 0.9 & 0.8 & 0.7 & 0.6 & 0.5 & 0.4 \\
\hline \multicolumn{7}{|l|}{$\rho_{12}$} \\
\hline 0.9 & 121 & 231 & 327 & 410 & 480 & 537 \\
\hline 0.8 & 196 & 425 & 626 & 799 & 945 & 1063 \\
\hline 0.7 & 223 & 581 & 896 & 1167 & 1395 & 1580 \\
\hline 0.6 & 200 & 699 & 1137 & 1514 & 1831 & 2088 \\
\hline 0.5 & & 780 & 1350 & 1841 & 2254 & 2588 \\
\hline 0.4 & & 823 & 1534 & 2147 & 2661 & 3078 \\
\hline 0.3 & & 827 & 1689 & 2432 & 3055 & 3560 \\
\hline 0.2 & & & 1816 & 2696 & 3435 & 4033 \\
\hline 0.1 & & & 1914 & 2939 & 3800 & 4497 \\
\hline 0 & & & 1983 & 3162 & 4152 & 4953 \\
\hline-0.1 & & & & 3364 & 4489 & 5399 \\
\hline-0.2 & & & & 3545 & 4812 & 5837 \\
\hline-0.3 & & & & 3706 & 5121 & 6266 \\
\hline-0.4 & & & & & 5416 & 6687 \\
\hline-0.5 & & & & & 5696 & 7098 \\
\hline-0.6 & & & & & & 7501 \\
\hline-0.7 & & & & & & 7894 \\
\hline
\end{tabular}




\section{PART 2. POSSIBLE RANGE OF THE INTER-CORRELATION BETWEEN TWO}

\section{MODELS}

Assume that the correlation coefficients produced by two models on a certain data set are $\rho_{1}$ and $\rho_{2}$, respectively. The inter-correlation coefficient between the results computed by these two models is $\rho_{12}$. While $\rho_{1}$ and $\rho_{2}$ may range from 0 to $1, \rho_{12}$ is in principle limited by the values of $\rho_{1}$ and $\rho_{2}$. It is because the following correlation matrix (Eq.S4) need to be a non-negative definite matrix:

$$
\mathbf{M}=\left[\begin{array}{ccc}
1 & \rho_{1} & \rho_{2} \\
\rho_{1} & 1 & \rho_{12} \\
\rho_{2} & \rho_{12} & 1
\end{array}\right]
$$

The expansion of $\boldsymbol{M}$ is:

$$
1+2 \rho_{1} \rho_{2} \rho_{12}-\rho_{1}{ }^{2}-\rho_{2}{ }^{2}-\rho_{12}{ }^{2} \geq 0
$$

Thus, the possible range of $\rho_{12}$ can be derived as:

$$
\rho_{1} \rho_{2}-\sqrt{\left(\rho_{1}{ }^{2}-1\right)\left(\rho_{2}{ }^{2}-1\right)} \leq \rho_{12} \leq \rho_{1} \rho_{2}+\sqrt{\left(\rho_{1}{ }^{2}-1\right)\left(\rho_{2}{ }^{2}-1\right)}
$$

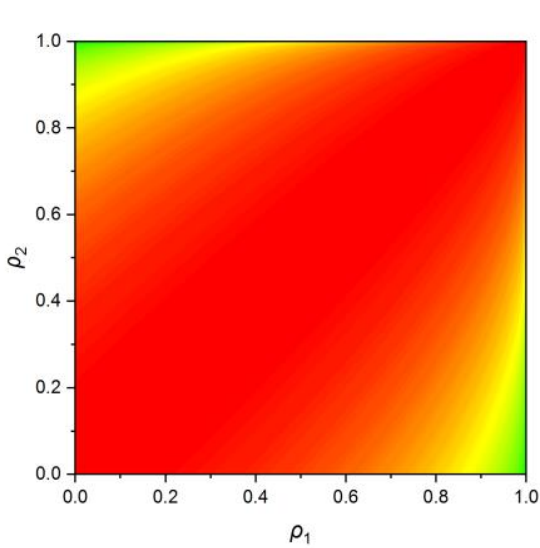

(a)

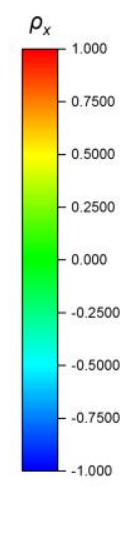

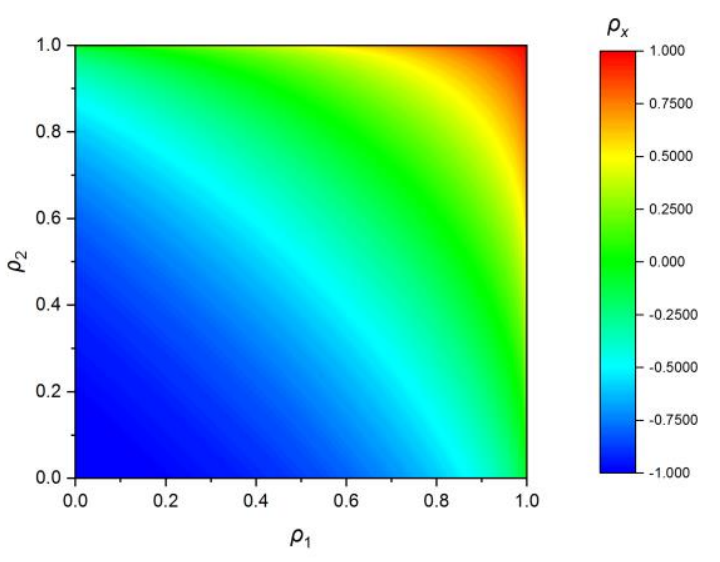

(b)

Figure S1. The possible range of $\rho_{12}$ as a function of $\rho_{l}$ and $\rho_{l}$. (a) The upper limit; (b) The lower limit. 


\section{PART 3. HYPOTHESIS TEST ON MOTHER SETS IN NORMAL DISTRIBUTION}

A total of 17 different configurations of $\rho_{\mathrm{A}}, \rho_{\mathrm{B}}$, and $\rho_{\mathrm{AB}}$ were considered (see Table 2 in the main text) for generating the mother sets in normal distribution. For each configuration, 100,000 virtual samples were generated by using the MASS module in the $R$ software.

The distributions of Carlson's test statistic and Dunn \& Clark's test statistic are shown below in Figure S2 and Figure S3, respectively. The results from the normality test of all distributions are summarized in Table S3-S8. One can see that Carlson's test statistic is not in normal distribution in almost all cases; while Dunn \& Clark's test statistic is always in normal distribution. For Dunn \& Clark's test statistic, the mean value and standard deviation of the distribution are very close to the theoretical value ( 0 and 1$)$ in all cases (Table S7). The type I error rates are also very close to the desired nominal significance level (e.g. 0.05) of a normal distribution (Table S8). 

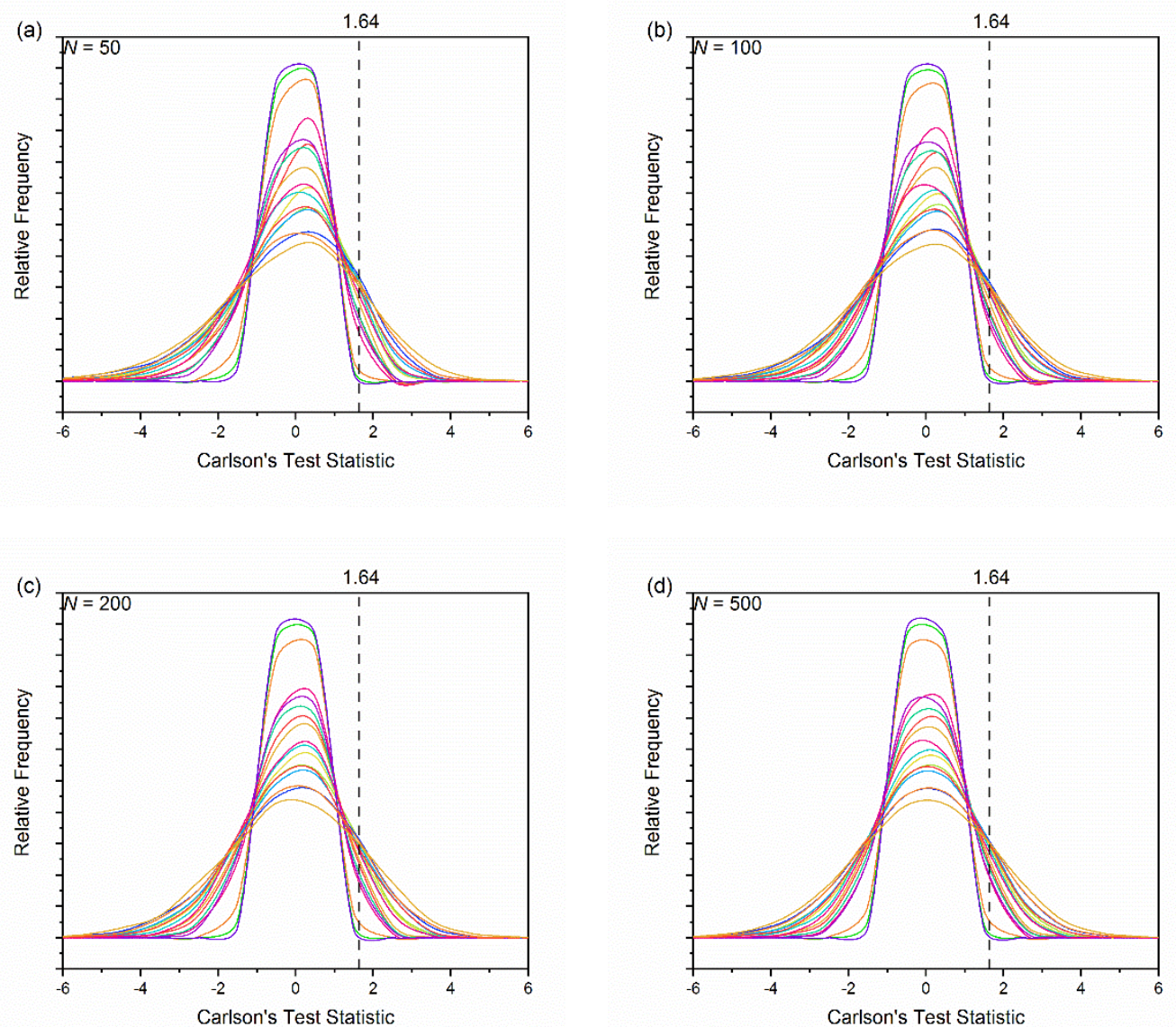

Figure S2. Distributions of Carlson's test statistic on four sample sizes assuming $\rho_{\mathrm{A}}=\rho_{\mathrm{B}}$. (a) $N=50$; (b) $N=100$; (c) $N=200$; (d) $N=500$. Distributions obtained on different mother sets are colored differently. The critical point (1.64) at the 95 percentile of a standard normal distribution is indicated on all figures. 
Table S3. Normality Test of Carlson's Test Statistic Derived from 17 Mother Sets in Normal Distribution

\begin{tabular}{cccccccccccr}
\hline $\begin{array}{c}\text { Mother } \\
\text { set }\end{array}$ & $\rho_{\mathrm{A}}$ & $\rho_{\mathrm{B}}$ & $\rho_{\mathrm{AB}}$ & \multicolumn{2}{c}{$\mathrm{N}=50$} & \multicolumn{2}{c}{$\mathrm{N}=100$} & \multicolumn{2}{c}{$\mathrm{N}=200$} & \multicolumn{2}{c}{$\mathrm{N}=500$} \\
\hline $1-1$ & 0.9 & 0.9 & 0.9 & 72.19 & $<2.20 \mathrm{E}-16$ & 27.56 & $<2.20 \mathrm{E}-16$ & 12.49 & $<2.20 \mathrm{E}-16$ & 4.58 & $2.32 \mathrm{E}-11$ \\
$1-2$ & 0.9 & 0.9 & 0.7 & 82.90 & $<2.20 \mathrm{E}-16$ & 35.16 & $<2.20 \mathrm{E}-16$ & 14.09 & $<2.20 \mathrm{E}-16$ & 6.98 & $<2.20 \mathrm{E}-16$ \\
$2-1$ & 0.7 & 0.7 & 0.9 & 16.17 & $<2.20 \mathrm{E}-16$ & 7.15 & $<2.20 \mathrm{E}-16$ & 3.58 & $6.23 \mathrm{E}-09$ & 0.87 & $2.59 \mathrm{E}-02$ \\
$2-2$ & 0.7 & 0.7 & 0.6 & 52.60 & $<2.20 \mathrm{E}-16$ & 25.74 & $<2.20 \mathrm{E}-16$ & 12.92 & $<2.20 \mathrm{E}-16$ & 5.49 & $1.56 \mathrm{E}-13$ \\
$2-3$ & 0.7 & 0.7 & 0.3 & 79.56 & $<2.20 \mathrm{E}-16$ & 35.00 & $<2.20 \mathrm{E}-16$ & 13.91 & $<2.20 \mathrm{E}-16$ & 6.75 & $<2.20 \mathrm{E}-16$ \\
$2-4$ & 0.7 & 0.7 & 0.0 & 80.98 & $<2.20 \mathrm{E}-16$ & 38.15 & $<2.20 \mathrm{E}-16$ & 16.85 & $<2.20 \mathrm{E}-16$ & 7.89 & $<2.20 \mathrm{E}-16$ \\
$3-1$ & 0.5 & 0.5 & 0.9 & 6.13 & $4.54 \mathrm{E}-15$ & 2.67 & $9.76 \mathrm{E}-07$ & 1.45 & $9.39 \mathrm{E}-04$ & 0.40 & $3.58 \mathrm{E}-01$ \\
$3-2$ & 0.5 & 0.5 & 0.6 & 22.40 & $<2.20 \mathrm{E}-16$ & 12.34 & $<2.20 \mathrm{E}-16$ & 4.09 & $3.53 \mathrm{E}-10$ & 2.18 & $1.60 \mathrm{E}-05$ \\
$3-3$ & 0.5 & 0.5 & 0.3 & 35.15 & $<2.20 \mathrm{E}-16$ & 15.98 & $<2.20 \mathrm{E}-16$ & 9.10 & $<2.20 \mathrm{E}-16$ & 4.08 & $3.77 \mathrm{E}-10$ \\
$3-4$ & 0.5 & 0.5 & 0.0 & 50.56 & $<2.20 \mathrm{E}-16$ & 22.50 & $<2.20 \mathrm{E}-16$ & 8.63 & $<2.20 \mathrm{E}-16$ & 4.22 & $1.75 \mathrm{E}-10$ \\
$3-5$ & 0.5 & 0.5 & -0.3 & 56.00 & $<2.20 \mathrm{E}-16$ & 23.58 & $<2.20 \mathrm{E}-16$ & 12.39 & $<2.20 \mathrm{E}-16$ & 5.66 & $6.10 \mathrm{E}-14$ \\
$4-1$ & 0.3 & 0.3 & 0.9 & 2.11 & $2.38 \mathrm{E}-05$ & 1.11 & $6.71 \mathrm{E}-03$ & 0.60 & $1.17 \mathrm{E}-01$ & 0.32 & $5.37 \mathrm{E}-01$ \\
$4-2$ & 0.3 & 0.3 & 0.6 & 7.08 & $<2.20 \mathrm{E}-16$ & 2.95 & $2.03 \mathrm{E}-07$ & 1.89 & $7.91 \mathrm{E}-05$ & 0.42 & $3.27 \mathrm{E}-01$ \\
$4-3$ & 0.3 & 0.3 & 0.3 & 12.15 & $<2.20 \mathrm{E}-16$ & 4.70 & $1.20 \mathrm{E}-11$ & 3.35 & $2.17 \mathrm{E}-08$ & 0.81 & $3.57 \mathrm{E}-02$ \\
$4-4$ & 0.3 & 0.3 & 0.0 & 19.16 & $<2.20 \mathrm{E}-16$ & 7.90 & $<2.20 \mathrm{E}-16$ & 2.58 & $1.69 \mathrm{E}-06$ & 1.53 & $6.25 \mathrm{E}-04$ \\
$4-5$ & 0.3 & 0.3 & -0.3 & 19.96 & $<2.20 \mathrm{E}-16$ & 7.63 & $<2.20 \mathrm{E}-16$ & 4.79 & $7.53 \mathrm{E}-12$ & 2.33 & $6.90 \mathrm{E}-06$ \\
$4-6$ & 0.3 & 0.3 & -0.6 & 23.30 & $<2.20 \mathrm{E}-16$ & 9.39 & $<2.20 \mathrm{E}-16$ & 4.06 & $4.36 \mathrm{E}-10$ & 2.38 & $5.02 \mathrm{E}-06$ \\
\hline
\end{tabular}

Table S4. Mean Values and Standard Deviations of Carlson's Test Statistic Derived from 17 Mother Sets in Normal Distribution

\begin{tabular}{cccccccccccc}
\hline $\begin{array}{c}\text { Mother } \\
\text { set }\end{array}$ & $\rho_{\mathrm{A}}$ & $\rho_{\mathrm{B}}$ & $\rho_{\mathrm{AB}}$ & \multicolumn{2}{c}{$\mathrm{N}=50$} & \multicolumn{2}{c}{$\mathrm{N}=100$} & \multicolumn{2}{c}{$\mathrm{N}=200$} & \multicolumn{2}{c}{$\mathrm{N}=500$} \\
\hline $1-1$ & 0.9 & 0.9 & 0.9 & -0.10 & 0.96 & -0.05 & 0.93 & -0.04 & 0.91 & -0.03 & 0.91 \\
$1-2$ & 0.9 & 0.9 & 0.7 & -0.15 & 1.12 & -0.10 & 1.09 & -0.08 & 1.06 & -0.05 & 1.06 \\
$2-1$ & 0.7 & 0.7 & 0.9 & -0.04 & 0.62 & -0.03 & 0.61 & -0.02 & 0.61 & -0.02 & 0.60 \\
$2-2$ & 0.7 & 0.7 & 0.6 & -0.14 & 1.18 & -0.10 & 1.14 & -0.08 & 1.12 & -0.05 & 1.12 \\
$2-3$ & 0.7 & 0.7 & 0.3 & -0.18 & 1.43 & -0.13 & 1.38 & -0.10 & 1.35 & -0.06 & 1.34 \\
$2-4$ & 0.7 & 0.7 & 0.0 & -0.23 & 1.55 & -0.15 & 1.47 & -0.11 & 1.43 & -0.08 & 1.43 \\
$3-1$ & 0.5 & 0.5 & 0.9 & -0.02 & 0.52 & -0.01 & 0.51 & -0.01 & 0.51 & -0.01 & 0.51 \\
$3-2$ & 0.5 & 0.5 & 0.6 & -0.07 & 1.01 & -0.06 & 1.01 & -0.05 & 0.98 & -0.02 & 0.99 \\
$3-3$ & 0.5 & 0.5 & 0.3 & -0.13 & 1.34 & -0.09 & 1.30 & -0.07 & 1.28 & -0.05 & 1.28 \\
$3-4$ & 0.5 & 0.5 & 0.0 & -0.16 & 1.56 & -0.11 & 1.51 & -0.08 & 1.48 & -0.05 & 1.48 \\
$3-5$ & 0.5 & 0.5 & -0.3 & -0.22 & 1.78 & -0.14 & 1.68 & -0.11 & 1.65 & -0.08 & 1.65 \\
$4-1$ & 0.3 & 0.3 & 0.9 & -0.01 & 0.47 & -0.01 & 0.47 & -0.01 & 0.46 & -0.01 & 0.46 \\
$4-2$ & 0.3 & 0.3 & 0.6 & -0.04 & 0.94 & -0.02 & 0.93 & -0.03 & 0.92 & -0.02 & 0.92 \\
$4-3$ & 0.3 & 0.3 & 0.3 & -0.08 & 1.25 & -0.04 & 1.22 & -0.04 & 1.22 & -0.04 & 1.21 \\
$4-4$ & 0.3 & 0.3 & 0.0 & -0.09 & 1.48 & -0.06 & 1.45 & -0.05 & 1.43 & -0.03 & 1.43 \\
$4-5$ & 0.3 & 0.3 & -0.3 & -0.14 & 1.72 & -0.09 & 1.65 & -0.07 & 1.62 & -0.05 & 1.63 \\
$4-6$ & 0.3 & 0.3 & -0.6 & -0.16 & 1.91 & -0.11 & 1.83 & -0.08 & 1.80 & -0.05 & 1.80 \\
\hline
\end{tabular}


Table S5. The Type I Error Rate of Carlson's Test Statistic Derived from 17 Mother Sets in Normal Distribution*

\begin{tabular}{ccccccccccccc}
\hline ID & $\rho_{\mathrm{A}}$ & $\rho_{\mathrm{B}}$ & $\rho_{\mathrm{AB}}$ & \multicolumn{2}{c}{$N=50$} & \multicolumn{2}{c}{$N=100$} & \multicolumn{2}{c}{$N=200$} & \multicolumn{2}{c}{$N=500$} \\
& & & & $\begin{array}{c}\text { one- } \\
\text { sided }\end{array}$ & $\begin{array}{c}\text { two- } \\
\text { sided }\end{array}$ & $\begin{array}{c}\text { one- } \\
\text { sided }\end{array}$ & $\begin{array}{c}\text { two- } \\
\text { sided }\end{array}$ & $\begin{array}{c}\text { one- } \\
\text { sided }\end{array}$ & $\begin{array}{c}\text { two- } \\
\text { sided }\end{array}$ & $\begin{array}{c}\text { one- } \\
\text { sided }\end{array}$ & $\begin{array}{c}\text { two- } \\
\text { sided }\end{array}$ \\
\hline $1-1$ & 0.9 & 0.9 & 0.9 & 0.0091 & 0.0439 & 0.019 & 0.0375 & 0.0229 & 0.0347 & 0.0268 & 0.0315 \\
$1-2$ & 0.9 & 0.9 & 0.7 & 0.0209 & 0.0736 & 0.0349 & 0.0665 & 0.0395 & 0.0629 & 0.0466 & 0.0618 \\
$2-1$ & 0.7 & 0.7 & 0.9 & 0.0006 & 0.0044 & 0.0013 & 0.0029 & 0.0013 & 0.0023 & 0.0017 & 0.0022 \\
$2-2$ & 0.7 & 0.7 & 0.6 & 0.0366 & 0.0823 & 0.0469 & 0.0791 & 0.0515 & 0.078 & 0.0593 & 0.079 \\
$2-3$ & 0.7 & 0.7 & 0.3 & 0.0666 & 0.1392 & 0.081 & 0.1441 & 0.0912 & 0.141 & 0.0953 & 0.1417 \\
$2-4$ & 0.7 & 0.7 & 0 & 0.081 & 0.166 & 0.0932 & 0.1671 & 0.0986 & 0.1626 & 0.1078 & 0.1683 \\
$3-1$ & 0.5 & 0.5 & 0.9 & 0.0001 & 0.0006 & 0.0002 & 0.0004 & 0.0002 & 0.0001 & 0.0007 & 0.0001 \\
$3-2$ & 0.5 & 0.5 & 0.6 & 0.0275 & 0.0513 & 0.0331 & 0.052 & 0.0359 & 0.0467 & 0.0426 & 0.0493 \\
$3-3$ & 0.5 & 0.5 & 0.3 & 0.0745 & 0.1256 & 0.0795 & 0.124 & 0.0814 & 0.1194 & 0.0867 & 0.1241 \\
$3-4$ & 0.5 & 0.5 & 0 & 0.1023 & 0.1788 & 0.1124 & 0.1842 & 0.1204 & 0.1835 & 0.1233 & 0.1791 \\
$3-5$ & 0.5 & 0.5 & -0.3 & 0.1299 & 0.2331 & 0.1334 & 0.2278 & 0.1379 & 0.2268 & 0.1461 & 0.234 \\
$4-1$ & 0.3 & 0.3 & 0.9 & 0 & 0 & 0.0001 & 0 & 0 & 0 & 0.0005 & 0 \\
$4-2$ & 0.3 & 0.3 & 0.6 & 0.0286 & 0.0382 & 0.0308 & 0.0358 & 0.0326 & 0.0331 & 0.0347 & 0.0331 \\
$4-3$ & 0.3 & 0.3 & 0.3 & 0.0728 & 0.1092 & 0.0778 & 0.1058 & 0.0774 & 0.1052 & 0.0831 & 0.1063 \\
$4-4$ & 0.3 & 0.3 & 0 & 0.1086 & 0.1709 & 0.1124 & 0.172 & 0.1187 & 0.1705 & 0.1189 & 0.1665 \\
$4-5$ & 0.3 & 0.3 & -0.3 & 0.141 & 0.2342 & 0.1408 & 0.2264 & 0.1414 & 0.2251 & 0.1499 & 0.2287 \\
$4-6$ & 0.3 & 0.3 & -0.6 & 0.1663 & 0.2835 & 0.1643 & 0.2759 & 0.1704 & 0.2788 & 0.1752 & 0.2744 \\
\hline
\end{tabular}

*: The significance level is 0.05 for both the one-sided and the two-sided tests. The critical values are 1.64 and $+/-1.96$, respectively. The test statistic is considered reasonable if the error rate is close to the nominal significance level. 

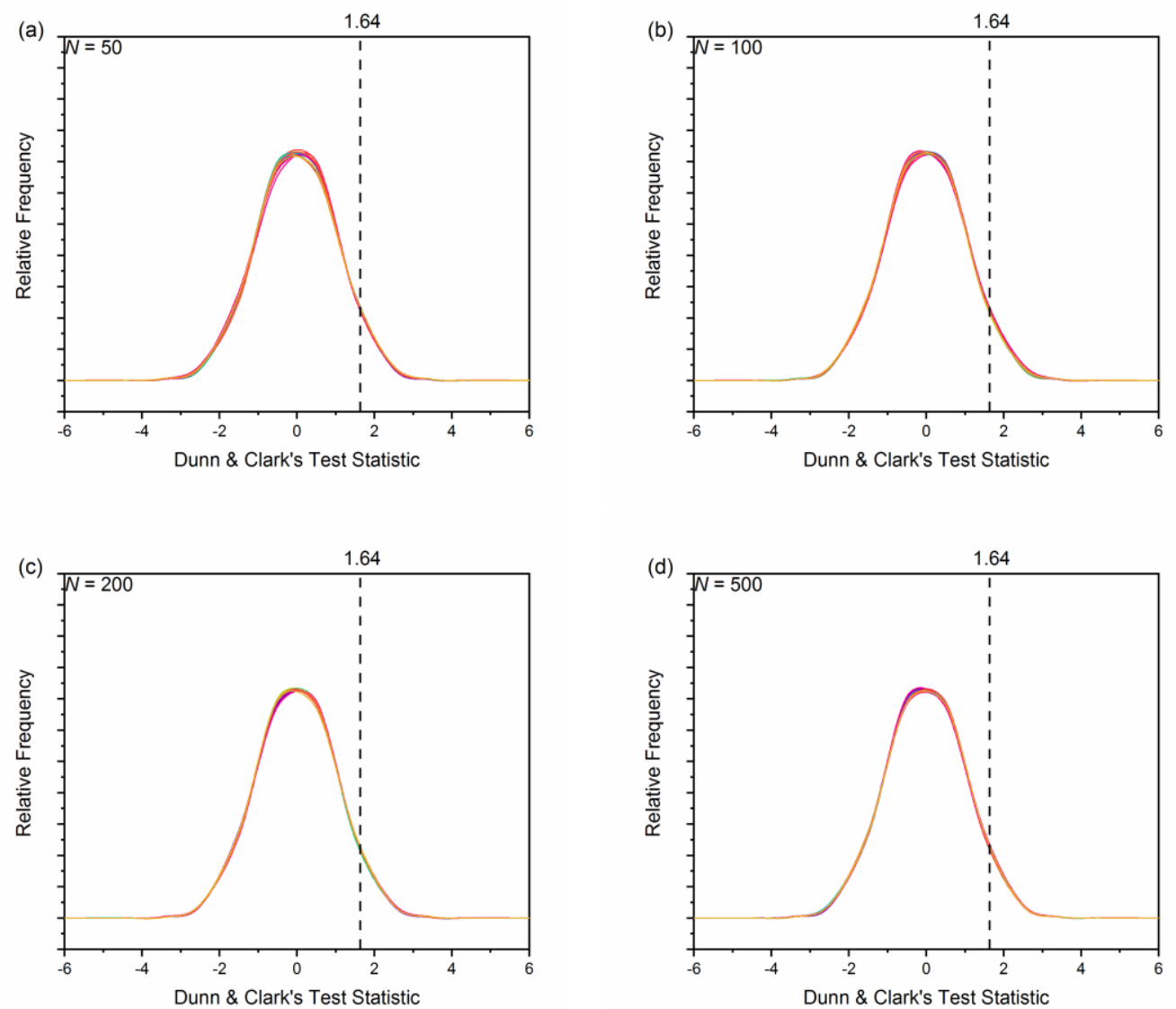

Figure S3. Distributions of Dunn \& Clark's test statistic on four sample sizes assuming $\rho_{\mathrm{A}}=$ $\rho_{\text {B. }}$ (a) $N=50$; (b) $N=100$; (c) $N=200$; (d) $N=500$. Distributions obtained on different mother sets are colored differently. The critical point (1.64) at the 95 percentile of a standard normal distribution is indicated on all figures. 
Table S6. Normality Test of Dunn \& Clark's Test Statistic Derived from 17 Mother Sets in Normal Distribution

\begin{tabular}{ccccccccccccc}
\hline $\begin{array}{c}\text { Mother } \\
\text { set }\end{array}$ & $\rho_{\mathrm{A}}$ & $\rho_{\mathrm{B}}$ & $\rho_{\mathrm{AB}}$ & \multicolumn{2}{c}{$\mathrm{N}=50$} & \multicolumn{2}{c}{$\mathrm{N}=100$} & \multicolumn{2}{c}{$\mathrm{N}=200$} & \multicolumn{2}{c}{$\mathrm{N}=500$} \\
\hline $1-1$ & 0.9 & 0.9 & 0.9 & 1.10 & 0.01 & 0.18 & 0.92 & 0.42 & 0.33 & 0.14 & 0.98 \\
$1-2$ & 0.9 & 0.9 & 0.7 & 0.53 & 0.17 & 0.28 & 0.66 & 0.50 & 0.21 & 0.23 & 0.80 \\
$2-1$ & 0.7 & 0.7 & 0.9 & 0.23 & 0.81 & 0.29 & 0.60 & 0.27 & 0.69 & 0.50 & 0.21 \\
$2-2$ & 0.7 & 0.7 & 0.6 & 0.84 & 0.03 & 0.19 & 0.90 & 0.21 & 0.86 & 0.27 & 0.69 \\
$2-3$ & 0.7 & 0.7 & 0.3 & 0.41 & 0.34 & 0.35 & 0.48 & 0.45 & 0.28 & 0.26 & 0.70 \\
$2-4$ & 0.7 & 0.7 & 0.0 & 0.42 & 0.32 & 0.35 & 0.47 & 0.48 & 0.24 & 0.21 & 0.87 \\
$3-1$ & 0.5 & 0.5 & 0.9 & 0.30 & 0.58 & 0.27 & 0.67 & 0.30 & 0.57 & 0.51 & 0.20 \\
$3-2$ & 0.5 & 0.5 & 0.6 & 0.31 & 0.56 & 0.35 & 0.47 & 0.21 & 0.85 & 0.12 & 0.99 \\
$3-3$ & 0.5 & 0.5 & 0.3 & 0.85 & 0.03 & 0.21 & 0.86 & 0.24 & 0.77 & 0.18 & 0.92 \\
$3-4$ & 0.5 & 0.5 & 0.0 & 0.37 & 0.43 & 0.32 & 0.53 & 0.44 & 0.29 & 0.27 & 0.67 \\
$3-5$ & 0.5 & 0.5 & -0.3 & 0.43 & 0.31 & 0.32 & 0.54 & 0.24 & 0.78 & 0.10 & 1.00 \\
$4-1$ & 0.3 & 0.3 & 0.9 & 0.41 & 0.35 & 0.28 & 0.64 & 0.31 & 0.55 & 0.45 & 0.27 \\
$4-2$ & 0.3 & 0.3 & 0.6 & 0.27 & 0.69 & 0.23 & 0.79 & 0.39 & 0.39 & 0.59 & 0.13 \\
$4-3$ & 0.3 & 0.3 & 0.3 & 0.21 & 0.85 & 0.31 & 0.56 & 0.57 & 0.14 & 0.94 & 0.02 \\
$4-4$ & 0.3 & 0.3 & 0.0 & 0.36 & 0.44 & 0.33 & 0.52 & 0.45 & 0.28 & 0.26 & 0.70 \\
$4-5$ & 0.3 & 0.3 & -0.3 & 0.36 & 0.45 & 0.34 & 0.50 & 0.20 & 0.89 & 0.11 & 0.99 \\
$4-6$ & 0.3 & 0.3 & -0.6 & 0.27 & 0.68 & 0.39 & 0.39 & 0.52 & 0.19 & 0.17 & 0.94 \\
\hline
\end{tabular}

Table S7. Mean Values and Standard Deviations of Dunn \& Clark's Test Statistic Derived from 17 Mother Sets in Normal Distribution

\begin{tabular}{cccccccccccc}
\hline $\begin{array}{c}\text { Mother } \\
\text { Set }\end{array}$ & $\rho_{\mathrm{A}}$ & $\rho_{\mathrm{B}}$ & $\rho_{\mathrm{AB}}$ & \multicolumn{2}{c}{$\mathrm{N}=50$} & \multicolumn{2}{c}{$\mathrm{N}=100$} & \multicolumn{2}{c}{$\mathrm{N}=200$} & \multicolumn{2}{c}{$\mathrm{N}=500$} \\
\hline $1-1$ & 0.9 & 0.9 & 0.9 & 0.01 & 1.02 & 0.03 & 1.01 & 0.01 & 1.00 & 0.00 & 1.00 \\
$1-2$ & 0.9 & 0.9 & 0.7 & 0.00 & 0.99 & 0.00 & 0.99 & -0.01 & 0.98 & 0.00 & 0.98 \\
$2-1$ & 0.7 & 0.7 & 0.9 & -0.01 & 0.99 & 0.00 & 0.99 & -0.01 & 0.99 & -0.01 & 0.99 \\
$2-2$ & 0.7 & 0.7 & 0.6 & -0.01 & 1.00 & 0.00 & 0.99 & -0.01 & 0.99 & -0.01 & 0.99 \\
$2-3$ & 0.7 & 0.7 & 0.3 & 0.00 & 0.99 & 0.00 & 0.99 & -0.01 & 0.98 & 0.00 & 0.98 \\
$2-4$ & 0.7 & 0.7 & 0.0 & -0.01 & 1.01 & 0.00 & 0.99 & -0.01 & 0.98 & -0.01 & 0.99 \\
$3-1$ & 0.5 & 0.5 & 0.9 & -0.01 & 0.99 & 0.00 & 0.99 & -0.01 & 0.99 & -0.01 & 0.99 \\
$3-2$ & 0.5 & 0.5 & 0.6 & 0.00 & 0.98 & -0.01 & 0.99 & -0.01 & 0.98 & 0.00 & 0.99 \\
$3-3$ & 0.5 & 0.5 & 0.3 & -0.01 & 1.00 & 0.00 & 0.99 & -0.01 & 0.99 & -0.01 & 0.99 \\
$3-4$ & 0.5 & 0.5 & 0.0 & 0.00 & 0.99 & 0.00 & 0.99 & 0.00 & 0.98 & 0.00 & 0.99 \\
$3-5$ & 0.5 & 0.5 & -0.3 & -0.01 & 1.01 & 0.00 & 0.99 & -0.01 & 0.99 & -0.01 & 1.00 \\
$4-1$ & 0.3 & 0.3 & 0.9 & -0.01 & 0.98 & 0.00 & 0.99 & -0.01 & 0.99 & -0.01 & 0.99 \\
$4-2$ & 0.3 & 0.3 & 0.6 & -0.01 & 0.99 & 0.00 & 0.99 & -0.01 & 0.99 & -0.01 & 0.99 \\
$4-3$ & 0.3 & 0.3 & 0.3 & -0.01 & 1.00 & 0.00 & 0.99 & -0.01 & 1.00 & -0.01 & 0.99 \\
$4-4$ & 0.3 & 0.3 & 0.0 & 0.00 & 0.99 & 0.00 & 0.99 & -0.01 & 0.98 & 0.00 & 0.99 \\
$4-5$ & 0.3 & 0.3 & -0.3 & -0.01 & 1.01 & 0.00 & 0.99 & -0.01 & 0.99 & -0.01 & 1.00 \\
$4-6$ & 0.3 & 0.3 & -0.6 & 0.00 & 1.01 & -0.01 & 1.00 & -0.01 & 0.99 & 0.00 & 1.00 \\
\hline
\end{tabular}


Table S8. The Type I Error Rate of Dunn \& Clark's Test Statistic Derived from 17 Mother Sets in Normal Distribution*

\begin{tabular}{lcccccccccccc}
\hline ID & $\rho_{\mathrm{A}}$ & $\rho_{\mathrm{B}}$ & $\rho_{\mathrm{AB}}$ & \multicolumn{2}{c}{$N=50$} & \multicolumn{2}{c}{$N=100$} & \multicolumn{2}{c}{$N=200$} & \multicolumn{2}{c}{$N=500$} \\
& & & & one-sided & $\begin{array}{c}\text { two- } \\
\text { twded }\end{array}$ & one-sided & $\begin{array}{c}\text { two- } \\
\text { sided }\end{array}$ & one-sided & $\begin{array}{c}\text { two- } \\
\text { sided }\end{array}$ & one-sided & $\begin{array}{c}\text { two- } \\
\text { sided }\end{array}$ \\
\hline $1-1$ & 0.9 & 0.9 & 0.9 & 0.0513 & 0.0557 & 0.055 & 0.0542 & 0.0533 & 0.0512 & 0.0511 & 0.0504 \\
$1-2$ & 0.9 & 0.9 & 0.7 & 0.0468 & 0.0486 & 0.0502 & 0.0492 & 0.0482 & 0.0467 & 0.0479 & 0.0463 \\
$2-1$ & 0.7 & 0.7 & 0.9 & 0.0492 & 0.0468 & 0.0482 & 0.049 & 0.0488 & 0.0458 & 0.0489 & 0.0464 \\
$2-2$ & 0.7 & 0.7 & 0.6 & 0.0476 & 0.0476 & 0.0479 & 0.0468 & 0.0472 & 0.0472 & 0.0471 & 0.0487 \\
$2-3$ & 0.7 & 0.7 & 0.3 & 0.0478 & 0.0486 & 0.0501 & 0.0478 & 0.0499 & 0.0464 & 0.0474 & 0.0454 \\
$2-4$ & 0.7 & 0.7 & 0 & 0.0499 & 0.0504 & 0.0488 & 0.0465 & 0.046 & 0.0459 & 0.051 & 0.0488 \\
$3-1$ & 0.5 & 0.5 & 0.9 & 0.0485 & 0.0459 & 0.0478 & 0.0476 & 0.0485 & 0.046 & 0.0488 & 0.0465 \\
$3-2$ & 0.5 & 0.5 & 0.6 & 0.0451 & 0.0453 & 0.0464 & 0.0473 & 0.0476 & 0.046 & 0.05 & 0.0487 \\
$3-3$ & 0.5 & 0.5 & 0.3 & 0.0493 & 0.0497 & 0.049 & 0.047 & 0.0451 & 0.0475 & 0.0463 & 0.0498 \\
$3-4$ & 0.5 & 0.5 & 0 & 0.0478 & 0.0468 & 0.0504 & 0.0484 & 0.0493 & 0.0474 & 0.047 & 0.0464 \\
$3-5$ & 0.5 & 0.5 & -0.3 & 0.0497 & 0.0519 & 0.0511 & 0.0473 & 0.0452 & 0.0461 & 0.0467 & 0.0477 \\
$4-1$ & 0.3 & 0.3 & 0.9 & 0.0477 & 0.0437 & 0.0483 & 0.0462 & 0.0476 & 0.0446 & 0.0482 & 0.0459 \\
$4-2$ & 0.3 & 0.3 & 0.6 & 0.0482 & 0.0481 & 0.0489 & 0.0478 & 0.0471 & 0.0482 & 0.0503 & 0.0471 \\
$4-3$ & 0.3 & 0.3 & 0.3 & 0.049 & 0.0493 & 0.0481 & 0.049 & 0.0473 & 0.0496 & 0.0504 & 0.05 \\
$4-4$ & 0.3 & 0.3 & 0 & 0.0483 & 0.0482 & 0.0502 & 0.0484 & 0.0503 & 0.0475 & 0.0479 & 0.0462 \\
$4-5$ & 0.3 & 0.3 & -0.3 & 0.0506 & 0.0527 & 0.0516 & 0.0491 & 0.0455 & 0.0484 & 0.0475 & 0.0472 \\
$4-6$ & 0.3 & 0.3 & -0.6 & 0.051 & 0.0526 & 0.0484 & 0.0508 & 0.0464 & 0.0488 & 0.0488 & 0.0488 \\
\hline
\end{tabular}

*: The significance level is 0.05 for both the one-sided and the two-sided tests. The critical values are 1.64 and $+/-1.96$, respectively. The test statistic is considered reasonable if the error rate is close to the nominal significance level. 


\section{PART 4. HYPOTHESIS TEST ON MOTHER SETS IN UNIFORM DISTR IBUTION}

The same set of the 17 different configurations of $\rho_{\mathrm{A}}, \rho_{\mathrm{B}}$, and $\rho_{\mathrm{AB}}$ listed in Table 2 in the main text were considered for generating the mother sets in uniform distribution. However, for the configuration ID 2-4 $\left(\rho_{A}=\rho_{B}=0.7, \rho_{A B}=0.0\right)$, the correlation matrix did not yield a positive value and therefore was not possible (see part 2 in this material). For the rest of 16 configurations, 100,000 virtual samples were generated by using the SimMultiCorrData module in the $R$ software.

The distributions of Carlson's test statistic and Dunn \& Clark's test statistic are shown below in Figure S4 and Figure S5, respectively. The results from the normality test of all distributions are summarized in Tables S9-S14. One can see that Carlson's test statistic is not in normal distribution in almost all cases (Table S9-S11). Dunn \& Clark's test statistic is still in normal distribution in most cases. However, by taking P-value $<0.05$ as the threshold, it is not in normal distribution in a few cases (Table S12). The mean value and standard deviation in some cases also depart from the theoretical values (i.e. 0 and 1), for example on mother set 1-1 and 1-2 (Table S13). In such a case, it means that the probability of rejecting the hypothesis is not the same as the area of the nominal rejection region at the given critical value of 1.64 in one-sided test. The type I error rates also depart somewhat from the desired nominal significance level (e.g. 0.05) of a normal distribution (Table S14). 

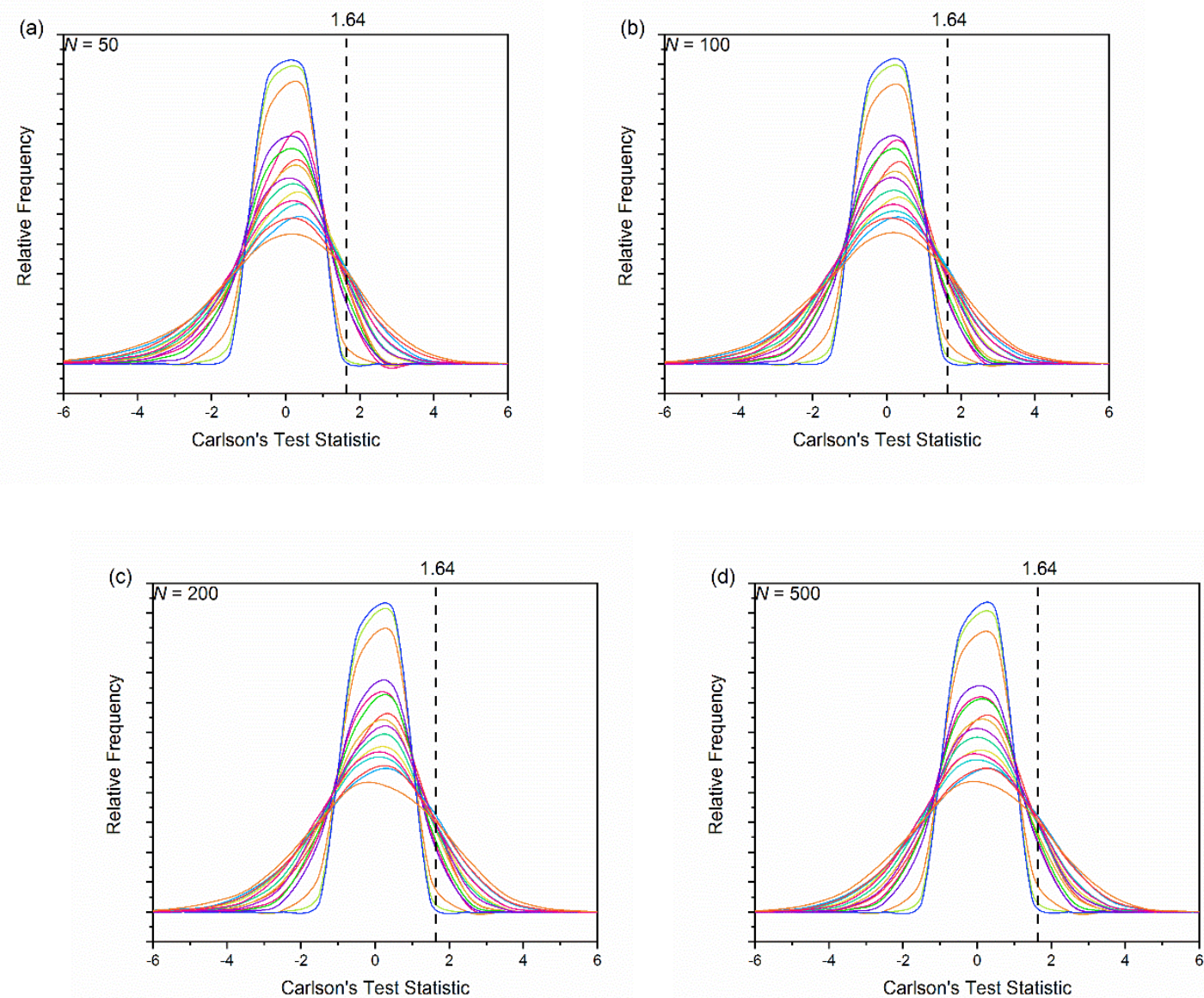

Figure S4. Distributions of Carlson's test statistic on four sample sizes assuming $\rho_{\mathrm{A}}=\rho_{\mathrm{B}}$. (a) $N=50$; (b) $N=100$; (c) $N=200$; (d) $N=500$. Distributions obtained on different mother sets are colored differently. The critical point (1.64) at the 95 percentile of a standard normal distribution is indicated on all figures. 
Table S9. Normality Test of Carlson's Test Statistic Derived from 16 Mother Sets in Uniform Distribution

\begin{tabular}{cccccccccccr}
\hline $\begin{array}{c}\text { Mother } \\
\text { set }\end{array}$ & $\rho_{\mathrm{A}}$ & $\rho_{\mathrm{B}}$ & \multicolumn{1}{c}{$\rho_{\mathrm{AB}}$} & \multicolumn{2}{c}{$\mathrm{N}=50$} & \multicolumn{2}{c}{$\mathrm{N}=100$} & \multicolumn{2}{c}{$\mathrm{N}=200$} & \multicolumn{2}{c}{$\mathrm{N}=500$} \\
& & & & \multicolumn{1}{c}{$\mathrm{A}$} & \multicolumn{1}{c}{ P-value } & \multicolumn{1}{c}{ A } & \multicolumn{1}{c}{ P-value } & \multicolumn{1}{c}{ A } & \multicolumn{1}{c}{ P-value } & \multicolumn{1}{c}{ A } & P-value \\
\hline $1-1$ & 0.9 & 0.9 & 0.9 & 82.76 & $<2.20 \mathrm{E}-16$ & 28.20 & $<2.20 \mathrm{E}-16$ & 15.59 & $<2.20 \mathrm{E}-16$ & 5.20 & $7.62 \mathrm{E}-13$ \\
$1-2$ & 0.9 & 0.9 & 0.7 & 90.81 & $<2.20 \mathrm{E}-16$ & 48.63 & $<2.20 \mathrm{E}-16$ & 28.29 & $<2.20 \mathrm{E}-16$ & 7.49 & $<2.20 \mathrm{E}-16$ \\
$2-1$ & 0.7 & 0.7 & 0.9 & 17.57 & $<2.20 \mathrm{E}-16$ & 8.16 & $<2.20 \mathrm{E}-16$ & 5.65 & $6.31 \mathrm{E}-14$ & 1.01 & $1.19 \mathrm{E}-02$ \\
$2-2$ & 0.7 & 0.7 & 0.6 & 64.01 & $<2.20 \mathrm{E}-16$ & 26.84 & $<2.20 \mathrm{E}-16$ & 14.95 & $<2.20 \mathrm{E}-16$ & 5.58 & $9.50 \mathrm{E}-14$ \\
$2-3$ & 0.7 & 0.7 & 0.3 & 87.52 & $<2.20 \mathrm{E}-16$ & 39.96 & $<2.20 \mathrm{E}-16$ & 20.27 & $<2.20 \mathrm{E}-16$ & 7.04 & $<2.20 \mathrm{E}-16$ \\
$3-1$ & 0.5 & 0.5 & 0.9 & 6.21 & $2.97 \mathrm{E}-15$ & 2.66 & $1.08 \mathrm{E}-06$ & 2.79 & $5.07 \mathrm{E}-07$ & 0.42 & $3.22 \mathrm{E}-01$ \\
$3-2$ & 0.5 & 0.5 & 0.6 & 21.75 & $<2.20 \mathrm{E}-16$ & 9.40 & $<2.20 \mathrm{E}-16$ & 7.20 & $<2.20 \mathrm{E}-16$ & 1.65 & $3.19 \mathrm{E}-04$ \\
$3-3$ & 0.5 & 0.5 & 0.3 & 40.14 & $<2.20 \mathrm{E}-16$ & 16.20 & $<2.20 \mathrm{E}-16$ & 8.85 & $<2.20 \mathrm{E}-16$ & 3.47 & $1.11 \mathrm{E}-08$ \\
$3-4$ & 0.5 & 0.5 & 0.0 & 51.01 & $<2.20 \mathrm{E}-16$ & 22.30 & $<2.20 \mathrm{E}-16$ & 10.89 & $<2.20 \mathrm{E}-16$ & 4.23 & $1.61 \mathrm{E}-10$ \\
$3-5$ & 0.5 & 0.5 & -0.3 & 57.37 & $<2.20 \mathrm{E}-16$ & 25.45 & $<2.20 \mathrm{E}-16$ & 13.62 & $<2.20 \mathrm{E}-16$ & 6.45 & $7.93 \mathrm{E}-16$ \\
$4-1$ & 0.3 & 0.3 & 0.9 & 2.62 & $1.35 \mathrm{E}-06$ & 0.93 & $1.83 \mathrm{E}-02$ & 1.58 & $4.59 \mathrm{E}-04$ & 0.40 & $3.71 \mathrm{E}-01$ \\
$4-2$ & 0.3 & 0.3 & 0.6 & 7.06 & $<2.20 \mathrm{E}-16$ & 2.71 & $8.18 \mathrm{E}-07$ & 3.22 & $4.55 \mathrm{E}-08$ & 0.56 & $1.47 \mathrm{E}-01$ \\
$4-3$ & 0.3 & 0.3 & 0.3 & 11.19 & $<2.20 \mathrm{E}-16$ & 4.10 & $3.42 \mathrm{E}-10$ & 4.51 & $3.55 \mathrm{E}-11$ & 1.14 & $5.52 \mathrm{E}-03$ \\
$4-4$ & 0.3 & 0.3 & 0.0 & 17.98 & $<2.20 \mathrm{E}-16$ & 6.82 & $<2.20 \mathrm{E}-16$ & 3.32 & $2.62 \mathrm{E}-08$ & 1.62 & $3.67 \mathrm{E}-04$ \\
$4-5$ & 0.3 & 0.3 & -0.3 & 20.61 & $<2.20 \mathrm{E}-16$ & 8.13 & $<2.20 \mathrm{E}-16$ & 5.65 & $6.56 \mathrm{E}-14$ & 2.63 & $1.25 \mathrm{E}-06$ \\
$4-6$ & 0.3 & 0.3 & -0.6 & 25.05 & $<2.20 \mathrm{E}-16$ & 11.04 & $<2.20 \mathrm{E}-16$ & 3.71 & $3.06 \mathrm{E}-09$ & 3.24 & $4.05 \mathrm{E}-08$ \\
\hline
\end{tabular}

Table S10. Mean Values and Standard Deviations of Carlson's Test Statistic Derived from 16 Mother Sets in Uniform Distribution

\begin{tabular}{cccccccccccc}
\hline $\begin{array}{c}\text { Mother } \\
\text { set }\end{array}$ & $\rho_{\mathrm{A}}$ & $\rho_{\mathrm{B}}$ & $\rho_{\mathrm{AB}}$ & \multicolumn{2}{c}{$\mathrm{N}=50$} & \multicolumn{2}{c}{$\mathrm{N}=100$} & \multicolumn{2}{c}{$\mathrm{N}=200$} & \multicolumn{2}{c}{$\mathrm{N}=500$} \\
\hline $1-1$ & 0.9 & 0.9 & 0.9 & -0.12 & 1.07 & -0.08 & 1.03 & -0.05 & 1.01 & -0.03 & 1.02 \\
$1-2$ & 0.9 & 0.9 & 0.7 & -0.16 & 1.26 & -0.08 & 1.22 & -0.02 & 1.21 & 0.06 & 1.18 \\
$2-1$ & 0.7 & 0.7 & 0.9 & -0.04 & 0.66 & -0.01 & 0.66 & 0.00 & 0.65 & 0.03 & 0.65 \\
$2-2$ & 0.7 & 0.7 & 0.6 & -0.14 & 1.26 & -0.11 & 1.23 & -0.08 & 1.21 & -0.05 & 1.20 \\
$2-3$ & 0.7 & 0.7 & 0.3 & -0.21 & 1.55 & -0.16 & 1.49 & -0.14 & 1.45 & -0.11 & 1.44 \\
$3-1$ & 0.5 & 0.5 & 0.9 & -0.01 & 0.53 & 0.00 & 0.53 & 0.01 & 0.53 & 0.03 & 0.53 \\
$3-2$ & 0.5 & 0.5 & 0.6 & -0.07 & 1.06 & -0.03 & 1.04 & -0.01 & 1.04 & 0.03 & 1.03 \\
$3-3$ & 0.5 & 0.5 & 0.3 & -0.13 & 1.38 & -0.10 & 1.35 & -0.09 & 1.33 & -0.06 & 1.33 \\
$3-4$ & 0.5 & 0.5 & 0.0 & -0.17 & 1.62 & -0.14 & 1.57 & -0.12 & 1.54 & -0.09 & 1.53 \\
$3-5$ & 0.5 & 0.5 & -0.3 & -0.21 & 1.79 & -0.10 & 1.69 & -0.04 & 1.67 & 0.01 & 1.66 \\
$4-1$ & 0.3 & 0.3 & 0.9 & 0.00 & 0.47 & 0.01 & 0.48 & 0.01 & 0.47 & 0.03 & 0.47 \\
$4-2$ & 0.3 & 0.3 & 0.6 & -0.03 & 0.95 & 0.00 & 0.95 & 0.01 & 0.94 & 0.04 & 0.94 \\
$4-3$ & 0.3 & 0.3 & 0.3 & -0.06 & 1.26 & -0.02 & 1.25 & 0.00 & 1.24 & 0.03 & 1.23 \\
$4-4$ & 0.3 & 0.3 & 0.0 & -0.09 & 1.50 & -0.08 & 1.48 & -0.08 & 1.45 & -0.05 & 1.45 \\
$4-5$ & 0.3 & 0.3 & -0.3 & -0.12 & 1.73 & -0.05 & 1.66 & -0.01 & 1.64 & 0.02 & 1.64 \\
$4-6$ & 0.3 & 0.3 & -0.6 & -0.15 & 1.93 & -0.09 & 1.85 & -0.07 & 1.82 & -0.06 & 1.81 \\
\hline
\end{tabular}


Table S11. The Type I Error Rate of Carlson's Test Statistic Derived from 16 Mother Sets in Uniform Distribution*

\begin{tabular}{ccccccccccccc}
\hline ID & $\rho_{\mathrm{A}}$ & $\rho_{\mathrm{B}}$ & $\rho_{\mathrm{AB}}$ & \multicolumn{2}{c}{$N=50$} & \multicolumn{2}{c}{$N=100$} & \multicolumn{2}{c}{$N=200$} & \multicolumn{2}{c}{$N=500$} \\
& & & & one-sided & $\begin{array}{c}\text { two- } \\
\text { sided }\end{array}$ & one-sided & $\begin{array}{c}\text { two- } \\
\text { sided }\end{array}$ & one-sided & $\begin{array}{c}\text { two- } \\
\text { sided }\end{array}$ & one-sided & $\begin{array}{c}\text { two- } \\
\text { sided }\end{array}$ \\
\hline $1-1$ & 0.9 & 0.9 & 0.9 & 0.017 & 0.0633 & 0.0303 & 0.0544 & 0.0332 & 0.052 & 0.0421 & 0.0538 \\
$1-2$ & 0.9 & 0.9 & 0.7 & 0.0345 & 0.0992 & 0.0532 & 0.0977 & 0.0666 & 0.0988 & 0.0821 & 0.0971 \\
$2-1$ & 0.7 & 0.7 & 0.9 & 0.0017 & 0.0072 & 0.0022 & 0.0045 & 0.0033 & 0.0039 & 0.0051 & 0.0029 \\
$2-2$ & 0.7 & 0.7 & 0.6 & 0.0487 & 0.1015 & 0.0601 & 0.104 & 0.065 & 0.1025 & 0.0703 & 0.1051 \\
$2-3$ & 0.7 & 0.7 & 0.3 & 0.0803 & 0.1642 & 0.0953 & 0.1706 & 0.0986 & 0.1709 & 0.1057 & 0.1673 \\
$3-1$ & 0.5 & 0.5 & 0.9 & 0.0004 & 0.0011 & 0.0004 & 0.0003 & 0.0005 & 0.0006 & 0.001 & 0.0005 \\
$3-2$ & 0.5 & 0.5 & 0.6 & 0.0354 & 0.061 & 0.0466 & 0.0598 & 0.0478 & 0.0557 & 0.0548 & 0.0582 \\
$3-3$ & 0.5 & 0.5 & 0.3 & 0.0793 & 0.1391 & 0.0871 & 0.1397 & 0.0882 & 0.1382 & 0.0933 & 0.1369 \\
$3-4$ & 0.5 & 0.5 & 0 & 0.1097 & 0.1934 & 0.1177 & 0.1984 & 0.1177 & 0.1974 & 0.1222 & 0.1946 \\
$3-5$ & 0.5 & 0.5 & -0.3 & 0.1327 & 0.2403 & 0.1426 & 0.2297 & 0.1518 & 0.232 & 0.1567 & 0.2354 \\
$4-1$ & 0.3 & 0.3 & 0.9 & 0.0002 & 0.0002 & 0.0002 & 0.0001 & 0 & 0 & 0.0004 & 0.0001 \\
$4-2$ & 0.3 & 0.3 & 0.6 & 0.0291 & 0.0402 & 0.0364 & 0.0406 & 0.037 & 0.0372 & 0.042 & 0.0377 \\
$4-3$ & 0.3 & 0.3 & 0.3 & 0.0801 & 0.1134 & 0.0855 & 0.1123 & 0.0876 & 0.1077 & 0.091 & 0.1109 \\
$4-4$ & 0.3 & 0.3 & 0 & 0.1121 & 0.1764 & 0.1155 & 0.1807 & 0.1138 & 0.177 & 0.119 & 0.1738 \\
$4-5$ & 0.3 & 0.3 & -0.3 & 0.143 & 0.2383 & 0.1485 & 0.2315 & 0.1545 & 0.23 & 0.1603 & 0.231 \\
$4-6$ & 0.3 & 0.3 & -0.6 & 0.1686 & 0.2811 & 0.1712 & 0.2804 & 0.1751 & 0.2764 & 0.1728 & 0.2753 \\
\hline
\end{tabular}

*: The significance level is 0.05 for both the one-sided and the two-sided tests. The critical values are 1.64 and $+/-1.96$, respectively. The test statistic is considered reasonable if the error rate is close to the nominal significance level. 

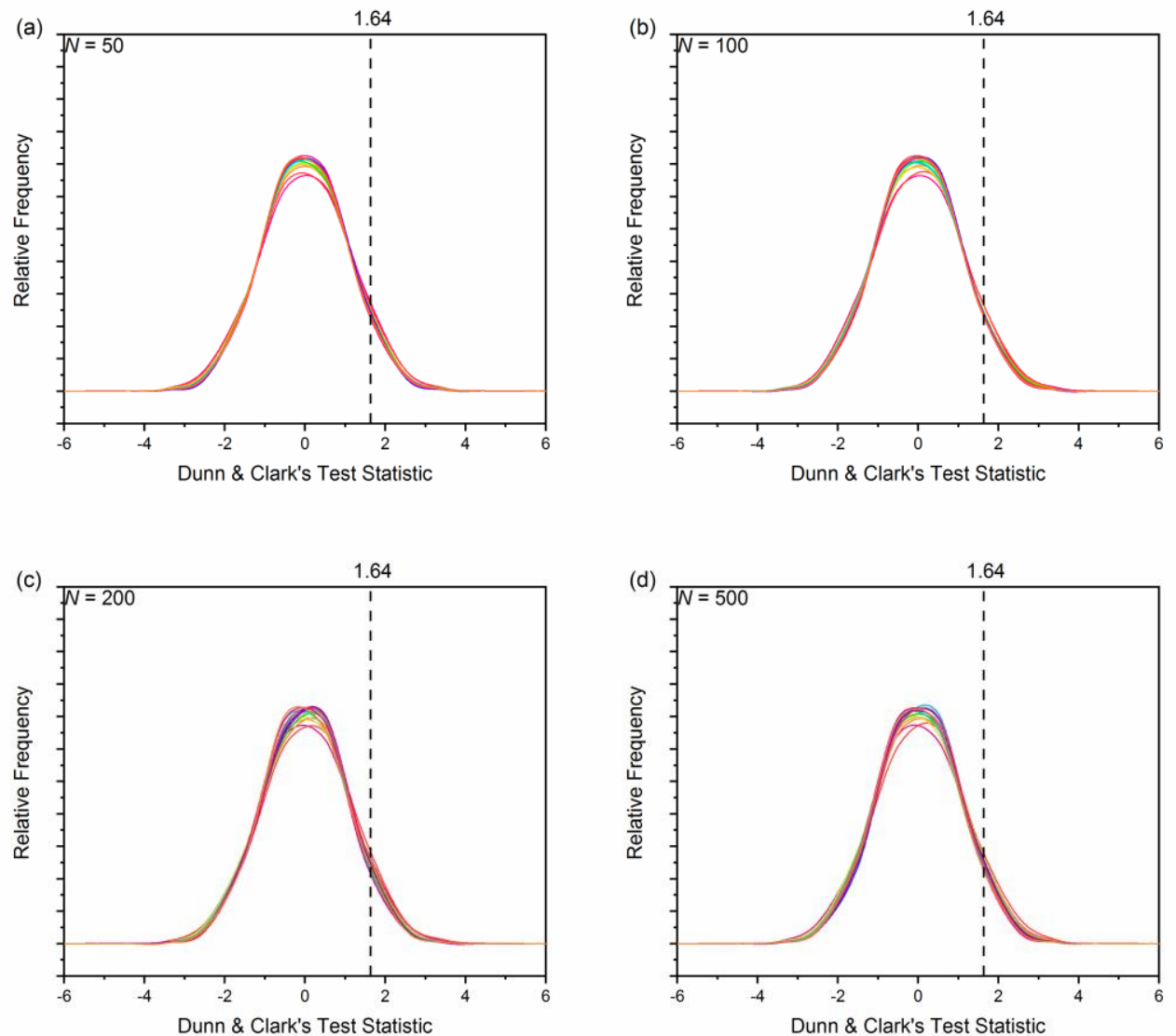

Figure S5. Distributions of Dunn \& Clark's test statistic on four sample sizes assuming $\rho_{\mathrm{A}}=$ $\rho_{\text {B. }}$ (a) $N=50$; (b) $N=100$; (c) $N=200$; (d) $N=500$. Distributions obtained on different mother sets are colored differently. The critical point (1.64) at the 95 percentile of a standard normal distribution is indicated on all figures. 
Table S12. Normality Test of Dunn \& Clark's Test Statistic Derived from 16 Mother Sets in Uniform Distribution

\begin{tabular}{cccccccccccc}
\hline $\begin{array}{c}\text { Mother } \\
\text { set }\end{array}$ & $\rho_{\mathrm{A}}$ & $\rho_{\mathrm{B}}$ & $\rho_{\mathrm{AB}}$ & \multicolumn{2}{c}{$\mathrm{N}=50$} & \multicolumn{2}{c}{$\mathrm{N}=100$} & \multicolumn{2}{c}{$\mathrm{N}=200$} & \multicolumn{2}{c}{$\mathrm{N}=500$} \\
\hline $1-1$ & 0.9 & 0.9 & 0.9 & 0.56 & 0.15 & 0.71 & 0.07 & 0.20 & 0.89 & 0.63 & 0.10 \\
$1-2$ & 0.9 & 0.9 & 0.7 & 0.44 & 0.29 & 0.39 & 0.38 & 0.62 & 0.11 & 0.40 & 0.36 \\
$2-1$ & 0.7 & 0.7 & 0.9 & 0.30 & 0.59 & 0.34 & 0.50 & 0.41 & 0.35 & 0.35 & 0.48 \\
$2-2$ & 0.7 & 0.7 & 0.6 & 0.49 & 0.23 & 0.21 & 0.85 & 0.25 & 0.73 & 0.35 & 0.47 \\
$2-3$ & 0.7 & 0.7 & 0.3 & 0.46 & 0.25 & 0.22 & 0.84 & 0.16 & 0.95 & 0.23 & 0.80 \\
$3-1$ & 0.5 & 0.5 & 0.9 & 0.44 & 0.29 & 0.25 & 0.74 & 0.51 & 0.19 & 0.41 & 0.34 \\
$3-2$ & 0.5 & 0.5 & 0.6 & 0.42 & 0.32 & 0.32 & 0.53 & 0.55 & 0.16 & 0.49 & 0.22 \\
$3-3$ & 0.5 & 0.5 & 0.3 & 0.52 & 0.19 & 0.26 & 0.70 & 0.26 & 0.70 & 0.35 & 0.47 \\
$3-4$ & 0.5 & 0.5 & 0.0 & 0.62 & 0.11 & 0.21 & 0.86 & 0.14 & 0.98 & 0.29 & 0.61 \\
$3-5$ & 0.5 & 0.5 & -0.3 & 0.78 & 0.04 & 0.40 & 0.37 & 0.27 & 0.67 & 0.23 & 0.79 \\
$4-1$ & 0.3 & 0.3 & 0.9 & 0.49 & 0.22 & 0.19 & 0.91 & 0.61 & 0.11 & 0.51 & 0.19 \\
$4-2$ & 0.3 & 0.3 & 0.6 & 0.36 & 0.45 & 0.31 & 0.54 & 0.58 & 0.13 & 0.49 & 0.23 \\
$4-3$ & 0.3 & 0.3 & 0.3 & 0.27 & 0.69 & 0.39 & 0.37 & 0.77 & 0.05 & 0.64 & 0.10 \\
$4-4$ & 0.3 & 0.3 & 0.0 & 0.48 & 0.23 & 0.21 & 0.85 & 0.19 & 0.90 & 0.32 & 0.53 \\
$4-5$ & 0.3 & 0.3 & -0.3 & 0.94 & 0.02 & 0.50 & 0.21 & 0.35 & 0.47 & 0.19 & 0.91 \\
$4-6$ & 0.3 & 0.3 & -0.6 & 0.53 & 0.17 & 0.30 & 0.57 & 0.64 & 0.10 & 0.22 & 0.84 \\
\hline
\end{tabular}

Table S13. Mean Values \& Standard Deviations of Dunn \& Clark's Test Statistic Derived from 16 Mother Sets in Uniform Distribution

\begin{tabular}{cccccccccccc}
\hline $\begin{array}{c}\text { Mother } \\
\text { set }\end{array}$ & $\rho_{\mathrm{A}}$ & $\rho_{\mathrm{B}}$ & $\rho_{\mathrm{AB}}$ & \multicolumn{2}{c}{$\mathrm{N}=50$} & \multicolumn{2}{c}{$\mathrm{N}=100$} & \multicolumn{2}{c}{$\mathrm{N}=200$} & \multicolumn{2}{c}{$\mathrm{N}=500$} \\
& & & & Mean & $\mathrm{SD}$ & Mean & $\mathrm{SD}$ & Mean & $\mathrm{SD}$ & Mean & $\mathrm{SD}$ \\
\hline $1-1$ & 0.9 & 0.9 & 0.9 & 0.03 & 1.12 & 0.02 & 1.12 & 0.02 & 1.10 & 0.01 & 1.11 \\
$1-2$ & 0.9 & 0.9 & 0.7 & 0.02 & 1.11 & 0.04 & 1.11 & 0.07 & 1.12 & 0.11 & 1.11 \\
$2-1$ & 0.7 & 0.7 & 0.9 & 0.01 & 1.06 & 0.03 & 1.06 & 0.04 & 1.06 & 0.06 & 1.07 \\
$2-2$ & 0.7 & 0.7 & 0.6 & 0.01 & 1.07 & 0.00 & 1.07 & -0.01 & 1.06 & -0.01 & 1.07 \\
$2-3$ & 0.7 & 0.7 & 0.3 & 0.00 & 1.06 & -0.01 & 1.06 & -0.03 & 1.05 & -0.03 & 1.06 \\
$3-1$ & 0.5 & 0.5 & 0.9 & 0.01 & 1.02 & 0.03 & 1.03 & 0.04 & 1.03 & 0.07 & 1.03 \\
$3-2$ & 0.5 & 0.5 & 0.6 & 0.01 & 1.03 & 0.03 & 1.03 & 0.03 & 1.03 & 0.05 & 1.03 \\
$3-3$ & 0.5 & 0.5 & 0.3 & 0.00 & 1.03 & -0.01 & 1.04 & -0.02 & 1.02 & -0.02 & 1.03 \\
$3-4$ & 0.5 & 0.5 & 0.0 & 0.00 & 1.03 & -0.01 & 1.03 & -0.03 & 1.02 & -0.03 & 1.02 \\
$3-5$ & 0.5 & 0.5 & -0.3 & 0.00 & 1.02 & 0.02 & 1.00 & 0.03 & 1.00 & 0.04 & 1.00 \\
$4-1$ & 0.3 & 0.3 & 0.9 & 0.02 & 0.99 & 0.03 & 1.00 & 0.04 & 1.00 & 0.07 & 1.00 \\
$4-2$ & 0.3 & 0.3 & 0.6 & 0.01 & 1.00 & 0.03 & 1.01 & 0.03 & 1.00 & 0.06 & 1.01 \\
$4-3$ & 0.3 & 0.3 & 0.3 & 0.00 & 1.01 & 0.02 & 1.01 & 0.02 & 1.01 & 0.04 & 1.01 \\
$4-4$ & 0.3 & 0.3 & 0.0 & 0.00 & 1.01 & -0.01 & 1.01 & -0.02 & 1.00 & -0.02 & 1.00 \\
$4-5$ & 0.3 & 0.3 & -0.3 & 0.00 & 1.02 & 0.02 & 1.00 & 0.03 & 1.00 & 0.04 & 1.00 \\
$4-6$ & 0.3 & 0.3 & -0.6 & 0.00 & 1.02 & 0.00 & 1.01 & 0.00 & 1.00 & -0.01 & 1.00 \\
\hline
\end{tabular}


Table S14. The Type I Error Rate of Dunn \& Clark's Test Statistic Derived from 16 Mother Sets in Uniform Distribution*

\begin{tabular}{ccccccccccccc}
\hline ID & $\rho_{\mathrm{A}}$ & $\rho_{\mathrm{B}}$ & $\rho_{\mathrm{AB}}$ & \multicolumn{2}{c}{$N=50$} & \multicolumn{2}{c}{$N=100$} & \multicolumn{2}{c}{$N=200$} & \multicolumn{2}{c}{$N=500$} \\
& & & & one-sided & $\begin{array}{c}\text { two- } \\
\text { sided }\end{array}$ & one-sided & $\begin{array}{c}\text { two- } \\
\text { sided }\end{array}$ & $\begin{array}{c}\text { one- } \\
\text { sided }\end{array}$ & two-sided & one-sided & $\begin{array}{c}\text { two- } \\
\text { sided }\end{array}$ \\
\hline $1-1$ & 0.9 & 0.9 & 0.9 & 0.0717 & 0.0788 & 0.0739 & 0.0773 & 0.0667 & 0.0751 & 0.0747 & 0.0785 \\
$1-2$ & 0.9 & 0.9 & 0.7 & 0.0747 & 0.0784 & 0.0745 & 0.0781 & 0.0754 & 0.0811 & 0.0834 & 0.0773 \\
$2-1$ & 0.7 & 0.7 & 0.9 & 0.0624 & 0.0625 & 0.0675 & 0.0664 & 0.0664 & 0.0647 & 0.0706 & 0.0677 \\
$2-2$ & 0.7 & 0.7 & 0.6 & 0.0632 & 0.0667 & 0.0631 & 0.0677 & 0.0586 & 0.0663 & 0.0583 & 0.0678 \\
$2-3$ & 0.7 & 0.7 & 0.3 & 0.0604 & 0.0626 & 0.0582 & 0.0643 & 0.0579 & 0.0628 & 0.0546 & 0.0661 \\
$3-1$ & 0.5 & 0.5 & 0.9 & 0.056 & 0.0521 & 0.0611 & 0.0573 & 0.0586 & 0.0554 & 0.0622 & 0.0573 \\
$3-2$ & 0.5 & 0.5 & 0.6 & 0.0575 & 0.0559 & 0.0598 & 0.0597 & 0.0577 & 0.0579 & 0.0624 & 0.0597 \\
$3-3$ & 0.5 & 0.5 & 0.3 & 0.0571 & 0.0588 & 0.0561 & 0.0585 & 0.054 & 0.0571 & 0.0511 & 0.0576 \\
$3-4$ & 0.5 & 0.5 & 0 & 0.0516 & 0.0572 & 0.0535 & 0.0555 & 0.0518 & 0.0552 & 0.0498 & 0.0545 \\
$3-5$ & 0.5 & 0.5 & -0.3 & 0.0573 & 0.0567 & 0.0539 & 0.0521 & 0.0539 & 0.0503 & 0.0568 & 0.0496 \\
$4-1$ & 0.3 & 0.3 & 0.9 & 0.0507 & 0.0466 & 0.0552 & 0.0524 & 0.0562 & 0.0501 & 0.0575 & 0.053 \\
$4-2$ & 0.3 & 0.3 & 0.6 & 0.0524 & 0.0488 & 0.0544 & 0.0535 & 0.0538 & 0.0519 & 0.0571 & 0.0525 \\
$4-3$ & 0.3 & 0.3 & 0.3 & 0.0527 & 0.0523 & 0.0543 & 0.0539 & 0.0513 & 0.0507 & 0.0572 & 0.0546 \\
$4-4$ & 0.3 & 0.3 & 0 & 0.0505 & 0.0532 & 0.0521 & 0.0507 & 0.0487 & 0.0503 & 0.0469 & 0.0485 \\
$4-5$ & 0.3 & 0.3 & -0.3 & 0.0555 & 0.0564 & 0.0535 & 0.0539 & 0.0504 & 0.0474 & 0.0531 & 0.0484 \\
$4-6$ & 0.3 & 0.3 & -0.6 & 0.0528 & 0.0564 & 0.0508 & 0.0527 & 0.0492 & 0.0506 & 0.0472 & 0.0505 \\
\hline
\end{tabular}

*: The significance level is 0.05 for both the one-sided and the two-sided tests. The critical values are 1.64 and $+/-1.96$, respectively. The test statistic is considered reasonable if the error rate is close to the nominal significance level. 


\section{PART 5. HYPOTHESIS TEST ON MOTHER SETS IN EXPONENTIAL DISTRIBUTION}

The same set of the 17 different configurations of $\rho_{\mathrm{A}}, \rho_{\mathrm{B}}$, and $\rho_{\mathrm{AB}}$ listed in Table 2 in the main text were considered for generating the mother sets in exponential distribution. However, for the configuration ID 2-4 $\left(\rho_{A}=\rho_{B}=0.7, \rho_{A B}=0.0\right), 3-5\left(\rho_{A}=\rho_{B}=0.5, \rho_{A B}=-0.3\right)$, and 4-6 $\left(\rho_{A}=\rho_{B}=0.3, \rho_{A B}=-0.6\right)$, the correlation matrix did not yield a positive value and therefore was not possible (see PART 2 in this material). For the rest of 14 configurations, 100,000 virtual samples were generated by using the SimMultiCorrData module in the $R$ software.

The distributions of Carlson's test statistic and Dunn \& Clark's test statistic are shown below in Figure S6 and Figure S7, respectively. The results from the normality test of all distributions are summarized in Tables S15-S20. Again, one can see that Carlson's test statistic is not in normal distribution in almost all cases (Table S15-S17). Here, Dunn \& Clark's test statistic is still in normal distribution in all cases. The mean values of those distributions are also very close to the theoretical value of 0 (Table S18). However, the standard deviations depart from the theoretical values of 1 significantly in all cases (Table S19). It means that the probability of rejecting the hypothesis is not the same as the area of the nominal rejection region at the given critical value of 1.64 in one-sided test. The type I error rates also depart more significantly from the desired nominal significance level (e.g. 0.05) of a normal distribution (Table S20). 

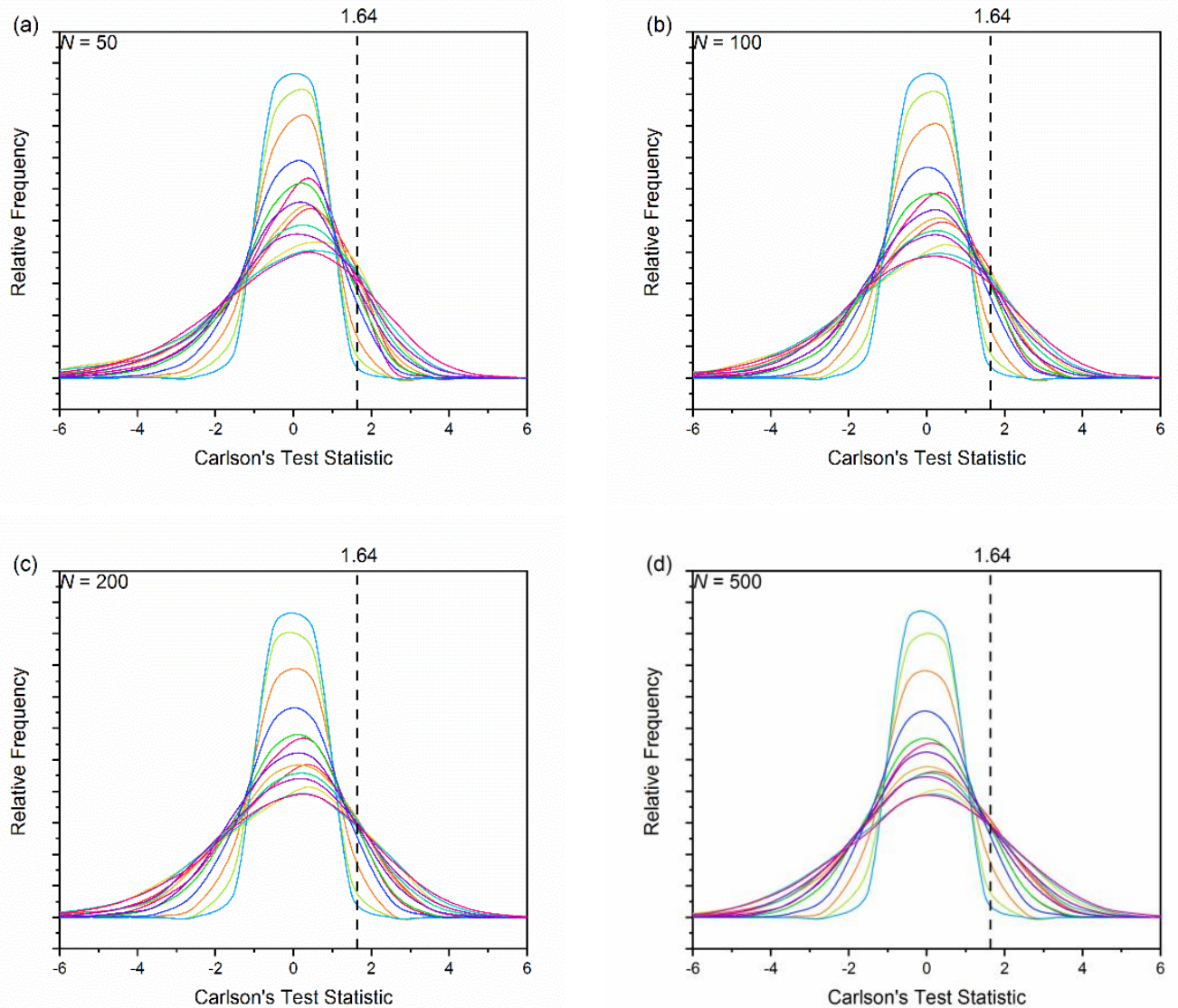

Figure S6. Distributions of Carlson's test statistic on four sample sizes assuming $\rho_{\mathrm{A}}=\rho_{\mathrm{B}}$. (a) $N=50$; (b) $N=100$; (c) $N=200$; (d) $N=500$. Distributions obtained on different mother sets are colored differently. The critical point (1.64) at the 95 percentile of a standard normal distribution is indicated on all figures. 
Table S15. Normality Test of Carlson's Test Statistic Derived from 14 Mother Sets in Exponential Distribution

\begin{tabular}{cccccccccccr}
\hline $\begin{array}{c}\text { Mother } \\
\text { Set }\end{array}$ & $\rho_{\mathrm{A}}$ & $\rho_{\mathrm{B}}$ & $\rho_{\mathrm{AB}}$ & \multicolumn{2}{c}{$N=50$} & \multicolumn{2}{c}{$N=100$} & \multicolumn{2}{c}{$N=200$} & \multicolumn{2}{c}{$N=500$} \\
\hline $1-1$ & 0.9 & 0.9 & 0.9 & 148.25 & $<2.20 \mathrm{E}-16$ & 62.72 & $<2.20 \mathrm{E}-16$ & 29.58 & $<2.20 \mathrm{E}-16$ & 17.03 & $<2.20 \mathrm{E}-16$ \\
$1-2$ & 0.9 & 0.9 & 0.7 & 182.02 & $<2.20 \mathrm{E}-16$ & 91.86 & $<2.20 \mathrm{E}-16$ & 37.42 & $<2.20 \mathrm{E}-16$ & 15.01 & $<2.20 \mathrm{E}-16$ \\
$2-1$ & 0.7 & 0.7 & 0.9 & 37.02 & $<2.20 \mathrm{E}-16$ & 15.20 & $<2.20 \mathrm{E}-16$ & 6.36 & $1.29 \mathrm{E}-15$ & 2.02 & $3.77 \mathrm{E}-05$ \\
$2-2$ & 0.7 & 0.7 & 0.6 & 117.56 & $<2.20 \mathrm{E}-16$ & 52.83 & $<2.20 \mathrm{E}-16$ & 19.86 & $<2.20 \mathrm{E}-16$ & 7.67 & $2.20 \mathrm{E}-16$ \\
$2-3$ & 0.7 & 0.7 & 0.3 & 172.75 & $<2.20 \mathrm{E}-16$ & 80.93 & $<2.20 \mathrm{E}-16$ & 33.18 & $<2.20 \mathrm{E}-16$ & 14.25 & $<2.20 \mathrm{E}-16$ \\
$3-1$ & 0.5 & 0.5 & 0.9 & 21.01 & $<2.20 \mathrm{E}-16$ & 9.67 & $<2.20 \mathrm{E}-16$ & 4.48 & $4.24 \mathrm{E}-11$ & 4.46 & $4.55 \mathrm{E}-11$ \\
$3-2$ & 0.5 & 0.5 & 0.6 & 47.94 & $<2.20 \mathrm{E}-16$ & 18.71 & $<2.20 \mathrm{E}-16$ & 9.03 & $<2.20 \mathrm{E}-16$ & 2.53 & $2.19 \mathrm{E}-06$ \\
$3-3$ & 0.5 & 0.5 & 0.3 & 78.92 & $<2.20 \mathrm{E}-16$ & 32.95 & $<2.20 \mathrm{E}-16$ & 11.62 & $<2.20 \mathrm{E}-16$ & 4.93 & $3.35 \mathrm{E}-12$ \\
$3-4$ & 0.5 & 0.5 & 0.0 & 105.70 & $<2.20 \mathrm{E}-16$ & 45.64 & $<2.20 \mathrm{E}-16$ & 18.66 & $<2.20 \mathrm{E}-16$ & 8.65 & $<2.20 \mathrm{E}-16$ \\
$4-1$ & 0.3 & 0.3 & 0.9 & 9.61 & $<2.20 \mathrm{E}-16$ & 2.40 & $4.49 \mathrm{E}-06$ & 0.90 & $2.23 \mathrm{E}-02$ & 0.37 & $4.35 \mathrm{E}-01$ \\
$4-2$ & 0.3 & 0.3 & 0.6 & 18.34 & $<2.20 \mathrm{E}-16$ & 5.93 & $1.40 \mathrm{E}-14$ & 2.53 & $2.19 \mathrm{E}-06$ & 0.64 & $9.44 \mathrm{E}-02$ \\
$4-3$ & 0.3 & 0.3 & 0.3 & 29.33 & $<2.20 \mathrm{E}-16$ & 10.29 & $<2.20 \mathrm{E}-16$ & 2.69 & $8.87 \mathrm{E}-07$ & 1.22 & $3.52 \mathrm{E}-03$ \\
$4-4$ & 0.3 & 0.3 & 0.0 & 38.79 & $<2.20 \mathrm{E}-16$ & 14.41 & $<2.20 \mathrm{E}-16$ & 5.00 & $2.27 \mathrm{E}-12$ & 2.91 & $2.60 \mathrm{E}-07$ \\
$4-5$ & 0.3 & 0.3 & -0.3 & 42.04 & $<2.20 \mathrm{E}-16$ & 19.43 & $<2.20 \mathrm{E}-16$ & 8.94 & $<2.20 \mathrm{E}-16$ & 4.76 & $8.66 \mathrm{E}-12$ \\
\hline
\end{tabular}

Table S16. Mean Values \& Standard Deviations of Carlson's Test Statistic Derived from 14 Mother Sets in Exponential Distribution

\begin{tabular}{cccccccccccc}
\hline $\begin{array}{c}\text { Mother } \\
\text { Set }\end{array}$ & $\rho_{\mathrm{A}}$ & $\rho_{\mathrm{B}}$ & $\rho_{\mathrm{AB}}$ & \multicolumn{2}{c}{$N=50$} & \multicolumn{2}{c}{$N=100$} & \multicolumn{2}{c}{$N=200$} & \multicolumn{2}{c}{$N=500$} \\
\hline $1-1$ & 0.9 & 0.9 & 0.9 & -0.25 & 1.51 & -0.18 & 1.45 & -0.18 & 1.42 & -0.16 & 1.43 \\
$1-2$ & 0.9 & 0.9 & 0.7 & -0.37 & 1.85 & -0.27 & 1.77 & -0.20 & 1.75 & -0.12 & 1.72 \\
$2-1$ & 0.7 & 0.7 & 0.9 & -0.09 & 0.88 & -0.06 & 0.89 & -0.05 & 0.89 & -0.05 & 0.89 \\
$2-2$ & 0.7 & 0.7 & 0.6 & -0.27 & 1.73 & -0.20 & 1.67 & -0.15 & 1.65 & -0.10 & 1.65 \\
$2-3$ & 0.7 & 0.7 & 0.3 & -0.43 & 2.25 & -0.31 & 2.12 & -0.23 & 2.08 & -0.14 & 2.04 \\
$3-1$ & 0.5 & 0.5 & 0.9 & -0.05 & 0.70 & -0.03 & 0.69 & -0.05 & 0.69 & -0.04 & 0.69 \\
$3-2$ & 0.5 & 0.5 & 0.6 & -0.15 & 1.37 & -0.11 & 1.35 & -0.10 & 1.35 & -0.10 & 1.35 \\
$3-3$ & 0.5 & 0.5 & 0.3 & -0.24 & 1.82 & -0.17 & 1.76 & -0.13 & 1.75 & -0.09 & 1.74 \\
$3-4$ & 0.5 & 0.5 & 0.0 & -0.34 & 2.24 & -0.24 & 2.13 & -0.19 & 2.11 & -0.12 & 2.08 \\
$4-1$ & 0.3 & 0.3 & 0.9 & -0.02 & 0.57 & -0.01 & 0.57 & -0.01 & 0.57 & -0.02 & 0.57 \\
$4-2$ & 0.3 & 0.3 & 0.6 & -0.07 & 1.14 & -0.05 & 1.14 & -0.05 & 1.14 & -0.06 & 1.14 \\
$4-3$ & 0.3 & 0.3 & 0.3 & -0.12 & 1.52 & -0.09 & 1.49 & -0.07 & 1.50 & -0.05 & 1.49 \\
$4-4$ & 0.3 & 0.3 & 0.0 & -0.17 & 1.86 & -0.12 & 1.80 & -0.11 & 1.80 & -0.08 & 1.79 \\
$4-5$ & 0.3 & 0.3 & -0.3 & -0.20 & 2.18 & -0.15 & 2.11 & -0.11 & 2.09 & -0.03 & 2.10 \\
\hline
\end{tabular}


Table S17. The Type I Error Rate of Carlson's Test Statistic Derived from 14 Mother Sets in Exponential Distribution*

\begin{tabular}{ccccccccccccc}
\hline ID & $\rho_{\mathrm{A}}$ & $\rho_{\mathrm{B}}$ & $\rho_{\mathrm{AB}}$ & \multicolumn{2}{c}{$N=50$} & \multicolumn{2}{c}{$N=100$} & \multicolumn{2}{c}{$N=200$} & \multicolumn{2}{c}{$N=500$} \\
& & & & one-sided & $\begin{array}{c}\text { two- } \\
\text { sided }\end{array}$ & one-sided & $\begin{array}{c}\text { two- } \\
\text { sided }\end{array}$ & one-sided & $\begin{array}{c}\text { two- } \\
\text { sided }\end{array}$ & one-sided & $\begin{array}{c}\text { two- } \\
\text { sided }\end{array}$ \\
\hline $1-1$ & 0.9 & 0.9 & 0.9 & 0.0504 & 0.1362 & 0.0786 & 0.1486 & 0.084 & 0.156 & 0.0951 & 0.1665 \\
$1-2$ & 0.9 & 0.9 & 0.7 & 0.0839 & 0.2024 & 0.1129 & 0.2252 & 0.1364 & 0.242 & 0.15 & 0.2493 \\
$2-1$ & 0.7 & 0.7 & 0.9 & 0.0115 & 0.0318 & 0.0182 & 0.0288 & 0.02 & 0.0296 & 0.0227 & 0.0287 \\
$2-2$ & 0.7 & 0.7 & 0.6 & 0.0953 & 0.1972 & 0.1153 & 0.2151 & 0.1282 & 0.2234 & 0.1409 & 0.2314 \\
$2-3$ & 0.7 & 0.7 & 0.3 & 0.1411 & 0.2828 & 0.1661 & 0.3059 & 0.1806 & 0.3309 & 0.1931 & 0.3309 \\
$3-1$ & 0.5 & 0.5 & 0.9 & 0.0033 & 0.0107 & 0.0051 & 0.0087 & 0.0065 & 0.0065 & 0.0052 & 0.0063 \\
$3-2$ & 0.5 & 0.5 & 0.6 & 0.0716 & 0.1342 & 0.0841 & 0.1351 & 0.0922 & 0.1416 & 0.095 & 0.144 \\
$3-3$ & 0.5 & 0.5 & 0.3 & 0.1236 & 0.2314 & 0.1403 & 0.2442 & 0.1494 & 0.257 & 0.1595 & 0.2598 \\
$3-4$ & 0.5 & 0.5 & 0 & 0.1684 & 0.3078 & 0.1821 & 0.3253 & 0.1919 & 0.3411 & 0.1967 & 0.3412 \\
$4-1$ & 0.3 & 0.3 & 0.9 & 0.002 & 0.0028 & 0.0018 & 0.0014 & 0.0014 & 0.0012 & 0.0019 & 0.0009 \\
$4-2$ & 0.3 & 0.3 & 0.6 & 0.0541 & 0.0825 & 0.0585 & 0.0828 & 0.0632 & 0.0832 & 0.064 & 0.0809 \\
$4-3$ & 0.3 & 0.3 & 0.3 & 0.1047 & 0.175 & 0.1151 & 0.1756 & 0.1214 & 0.1848 & 0.1271 & 0.1941 \\
$4-4$ & 0.3 & 0.3 & 0 & 0.1493 & 0.2521 & 0.1586 & 0.2605 & 0.1598 & 0.2715 & 0.1684 & 0.27 \\
$4-5$ & 0.3 & 0.3 & -0.3 & 0.1926 & 0.3347 & 0.1926 & 0.3296 & 0.2017 & 0.3342 & 0.2122 & 0.3453 \\
\hline
\end{tabular}

*: The significance level is 0.05 for both the one-sided and the two-sided tests. The critical values are 1.64 and $+/-1.96$, respectively. The test statistic is considered reasonable if the error rate is close to the nominal significance level. 

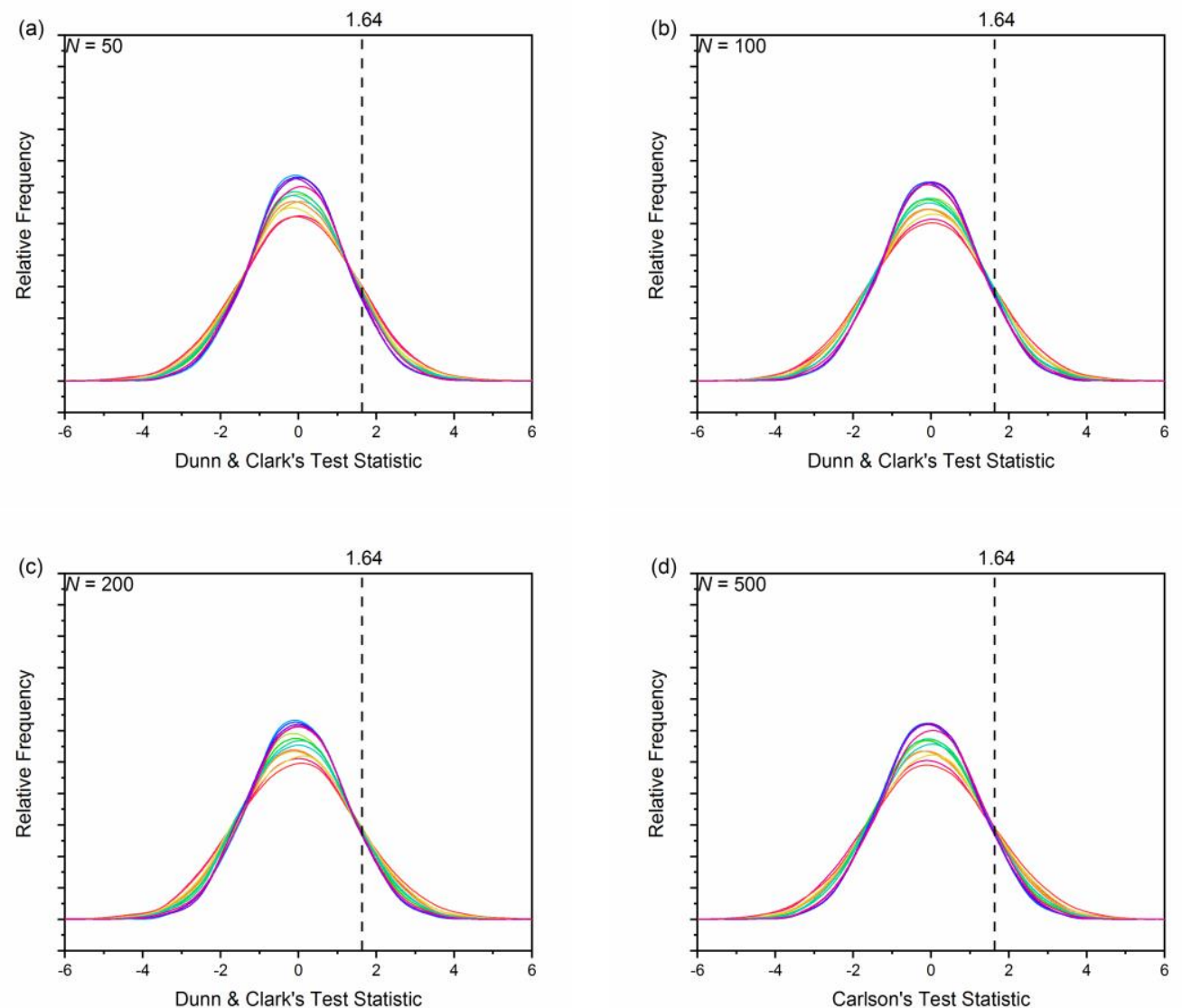

Figure S7. Distributions of Dunn \& Clark's test statistic on four sample sizes assuming $\rho_{\mathrm{A}}=$ $\rho_{\text {B. }}$ (a) $N=50$; (b) $N=100$; (c) $N=200$; (d) $N=500$. Distributions obtained on different mother sets are colored differently. The critical point (1.64) at the 95 percentile of a standard normal distribution is indicated on all figures. 
Table S18. Normality Test of Dunn \& Clark's Test Statistic Derived from 14 Mother Sets in Exponential Distribution

\begin{tabular}{ccccccccccccc}
\hline $\begin{array}{c}\text { Mother } \\
\text { Set }\end{array}$ & $\rho_{\mathrm{A}}$ & $\rho_{\mathrm{B}}$ & $\rho_{\mathrm{AB}}$ & \multicolumn{2}{c}{$N=50$} & \multicolumn{2}{c}{$N=100$} & \multicolumn{2}{c}{$N=200$} & \multicolumn{2}{c}{$N=500$} \\
\hline $1-1$ & 0.9 & 0.9 & 0.9 & 0.85 & 0.61 & 0.11 & 0.83 & 0.03 & 0.35 & 0.46 & 0.63 \\
$1-2$ & 0.9 & 0.9 & 0.7 & 0.91 & 0.91 & 0.02 & 0.23 & 0.81 & 0.90 & 0.02 & 0.30 \\
$2-1$ & 0.7 & 0.7 & 0.9 & 0.69 & 0.69 & 0.07 & 0.26 & 0.71 & 0.37 & 0.43 & 0.43 \\
$2-2$ & 0.7 & 0.7 & 0.6 & 0.63 & 0.65 & 0.09 & 0.18 & 0.92 & 0.72 & 0.06 & 0.47 \\
$2-3$ & 0.7 & 0.7 & 0.3 & 0.90 & 0.90 & 0.02 & 0.25 & 0.73 & 0.79 & 0.04 & 0.29 \\
$3-1$ & 0.5 & 0.5 & 0.9 & 0.26 & 0.25 & 0.74 & 0.43 & 0.30 & 0.63 & 0.10 & 1.14 \\
$3-2$ & 0.5 & 0.5 & 0.6 & 0.49 & 0.49 & 0.22 & 0.35 & 0.49 & 0.22 & 0.83 & 0.25 \\
$3-3$ & 0.5 & 0.5 & 0.3 & 0.80 & 0.80 & 0.04 & 0.21 & 0.86 & 0.63 & 0.10 & 0.24 \\
$3-4$ & 0.5 & 0.5 & 0.0 & 1.00 & 1.00 & 0.01 & 0.34 & 0.51 & 0.49 & 0.21 & 0.31 \\
$4-1$ & 0.3 & 0.3 & 0.9 & 0.55 & 0.54 & 0.17 & 0.24 & 0.78 & 0.25 & 0.76 & 0.53 \\
$4-2$ & 0.3 & 0.3 & 0.6 & 0.63 & 0.62 & 0.11 & 0.24 & 0.79 & 0.18 & 0.91 & 0.34 \\
$4-3$ & 0.3 & 0.3 & 0.3 & 0.65 & 0.72 & 0.06 & 0.21 & 0.86 & 0.61 & 0.11 & 0.33 \\
$4-4$ & 0.3 & 0.3 & 0.0 & 1.03 & 1.03 & 0.01 & 0.31 & 0.56 & 0.31 & 0.57 & 0.43 \\
$4-5$ & 0.3 & 0.3 & -0.3 & 0.16 & 0.16 & 0.94 & 0.63 & 0.10 & 0.32 & 0.54 & 0.33 \\
\hline
\end{tabular}

Table S19. Mean Values and Standard Deviations of Dunn \& Clark's Test Statistic Derived from 14 Mother Sets in Exponential Distribution

\begin{tabular}{cccccccccccc}
\hline $\begin{array}{c}\text { Mother } \\
\text { Set }\end{array}$ & $\rho_{\mathrm{A}}$ & $\rho_{\mathrm{B}}$ & $\rho_{\mathrm{AB}}$ & \multicolumn{2}{c}{$N=50$} & \multicolumn{2}{c}{$N=100$} & \multicolumn{2}{c}{$N=200$} & \multicolumn{2}{c}{$N=500$} \\
\hline $1-1$ & 0.9 & 0.9 & 0.9 & -0.01 & 1.49 & 0.00 & 1.52 & -0.06 & 1.52 & -0.08 & 1.54 \\
$1-2$ & 0.9 & 0.9 & 0.7 & -0.02 & 1.50 & -0.02 & 1.55 & -0.01 & 1.58 & 0.00 & 1.59 \\
$2-1$ & 0.7 & 0.7 & 0.9 & -0.01 & 1.37 & -0.02 & 1.41 & -0.02 & 1.43 & -0.04 & 1.45 \\
$2-2$ & 0.7 & 0.7 & 0.6 & -0.01 & 1.38 & -0.02 & 1.42 & -0.02 & 1.44 & -0.02 & 1.45 \\
$2-3$ & 0.7 & 0.7 & 0.3 & -0.02 & 1.43 & -0.02 & 1.46 & -0.02 & 1.49 & -0.01 & 1.49 \\
$3-1$ & 0.5 & 0.5 & 0.9 & -0.02 & 1.30 & -0.01 & 1.32 & -0.05 & 1.33 & -0.06 & 1.34 \\
$3-2$ & 0.5 & 0.5 & 0.6 & -0.02 & 1.29 & -0.02 & 1.31 & -0.03 & 1.33 & -0.06 & 1.34 \\
$3-3$ & 0.5 & 0.5 & 0.3 & -0.01 & 1.30 & -0.02 & 1.32 & -0.02 & 1.34 & -0.02 & 1.35 \\
$3-4$ & 0.5 & 0.5 & 0.0 & -0.02 & 1.34 & -0.02 & 1.36 & -0.02 & 1.38 & -0.02 & 1.38 \\
$4-1$ & 0.3 & 0.3 & 0.9 & -0.01 & 1.16 & -0.01 & 1.19 & -0.01 & 1.20 & -0.03 & 1.21 \\
$4-2$ & 0.3 & 0.3 & 0.6 & -0.01 & 1.18 & -0.01 & 1.20 & -0.02 & 1.21 & -0.04 & 1.22 \\
$4-3$ & 0.3 & 0.3 & 0.3 & -0.01 & 1.18 & -0.01 & 1.20 & -0.02 & 1.21 & -0.02 & 1.22 \\
$4-4$ & 0.3 & 0.3 & 0.0 & -0.01 & 1.21 & -0.02 & 1.21 & -0.02 & 1.23 & -0.02 & 1.23 \\
$4-5$ & 0.3 & 0.3 & -0.3 & 0.00 & 1.24 & -0.01 & 1.25 & -0.01 & 1.25 & 0.02 & 1.28 \\
\hline
\end{tabular}


Table S20. The Type I Error Rate of Dunn \& Clark's Test Statistic Derived from 14 Mother Sets in Exponential Distribution *

\begin{tabular}{ccccccccccccc}
\hline ID & $\rho_{\mathrm{A}}$ & $\rho_{\mathrm{B}}$ & $\rho_{\mathrm{AB}}$ & \multicolumn{2}{c}{$\begin{array}{c}N=50 \\
\text { one- } \\
\text { sided }\end{array}$} & $\begin{array}{c}\text { two- } \\
\text { sided }\end{array}$ & one-sided & $\begin{array}{c}\text { two- } \\
\text { sided }\end{array}$ & one-sided & $\begin{array}{c}\text { two- } \\
\text { sided }\end{array}$ & one-sided & $\begin{array}{c}\text { two- } \\
\text { sided }\end{array}$ \\
\hline $1-1$ & 0.9 & 0.9 & 0.9 & 0.1332 & 0.1867 & 0.1359 & 0.1958 & 0.1958 & 0.1958 & 0.1309 & 0.2067 \\
$1-2$ & 0.9 & 0.9 & 0.7 & 0.1264 & 0.1878 & 0.1391 & 0.2039 & 0.2039 & 0.2039 & 0.1515 & 0.2204 \\
$2-1$ & 0.7 & 0.7 & 0.9 & 0.1123 & 0.1534 & 0.119 & 0.1632 & 0.1632 & 0.1632 & 0.1218 & 0.1777 \\
$2-2$ & 0.7 & 0.7 & 0.6 & 0.1128 & 0.1541 & 0.1163 & 0.1666 & 0.1666 & 0.1666 & 0.1273 & 0.1792 \\
$2-3$ & 0.7 & 0.7 & 0.3 & 0.1162 & 0.1639 & 0.1223 & 0.178 & 0.178 & 0.178 & 0.1349 & 0.1923 \\
$3-1$ & 0.5 & 0.5 & 0.9 & 0.0999 & 0.1321 & 0.1021 & 0.1392 & 0.1392 & 0.1392 & 0.0963 & 0.1412 \\
$3-2$ & 0.5 & 0.5 & 0.6 & 0.098 & 0.1274 & 0.1022 & 0.1351 & 0.1351 & 0.1351 & 0.1032 & 0.1435 \\
$3-3$ & 0.5 & 0.5 & 0.3 & 0.0971 & 0.131 & 0.1016 & 0.137 & 0.137 & 0.137 & 0.1115 & 0.1497 \\
$3-4$ & 0.5 & 0.5 & 0 & 0.1028 & 0.1398 & 0.1069 & 0.1422 & 0.1422 & 0.1422 & 0.116 & 0.1549 \\
$4-1$ & 0.3 & 0.3 & 0.9 & 0.0765 & 0.0936 & 0.0815 & 0.0964 & 0.0964 & 0.0964 & 0.0835 & 0.1026 \\
$4-2$ & 0.3 & 0.3 & 0.6 & 0.0782 & 0.0976 & 0.0817 & 0.1011 & 0.1011 & 0.1011 & 0.0822 & 0.104 \\
$4-3$ & 0.3 & 0.3 & 0.3 & 0.0784 & 0.0991 & 0.0809 & 0.1033 & 0.1033 & 0.1033 & 0.0908 & 0.1078 \\
$4-4$ & 0.3 & 0.3 & 0 & 0.0808 & 0.104 & 0.0829 & 0.1021 & 0.1021 & 0.1021 & 0.0901 & 0.1139 \\
$4-5$ & 0.3 & 0.3 & -0.3 & 0.0913 & 0.1129 & 0.0923 & 0.1176 & 0.1176 & 0.1176 & 0.1007 & 0.1231 \\
\hline
\end{tabular}

*: The significance level is 0.05 for both the one-sided and the two-sided tests. The critical values are 1.64 and $+/-1.96$, respectively. The test statistic is considered reasonable if the error rate is close to the nominal significance level. 


\section{PART 6. ANALYSIS OF THE PEARSON CORRELATION COEFFICIENTS OBTAINED ON THE CASF-2013 BENCHMARK}

Table S21. Pearson correlation coefficients produced by all scoring functions as well as their inter-correlation coefficients obtained in the scoring power test in CASF-2013

\begin{tabular}{|c|c|c|c|c|c|c|c|c|c|c|c|c|c|c|c|c|c|c|c|c|c|c|c|}
\hline Rank & Scoring function & $R^{a}$ & $\# 1$ & \#2 & \#3 & \#4 & $\# 5$ & \#6 & $\# 7$ & $\# 8$ & $\# 9$ & $\# 10$ & $\# 11$ & $\# 12$ & $\# 13$ & \#14 & $\# 15$ & $\# 16$ & $\# 17$ & \#18 & $\# 19$ & $\# 20$ & \#21 \\
\hline 1 & X-ScoreHM & 0.614 & $1^{b}$ & 0.808 & 0.771 & 0.697 & 0.781 & 0.707 & 0.607 & 0.691 & 0.695 & 0.590 & 0.639 & 0.573 & 0.461 & 0.627 & 0.636 & 0.585 & 0.440 & 0.338 & 0.310 & 0.094 & 0.108 \\
\hline 2 & $\triangle \mathrm{SAS}$ & 0.606 & 0.808 & 1 & 0.615 & 0.818 & 0.880 & 0.820 & 0.739 & 0.759 & 0.594 & 0.790 & 0.825 & 0.556 & 0.607 & 0.657 & 0.720 & 0.537 & 0.490 & 0.461 & 0.270 & 0.353 & 0.287 \\
\hline 3 & ChemScore@SYBYL & 0.592 & 0.771 & 0.615 & 1 & 0.776 & 0.657 & 0.692 & 0.553 & 0.599 & 0.899 & 0.541 & 0.555 & 0.774 & 0.701 & 0.549 & 0.523 & 0.614 & 0.668 & 0.272 & 0.395 & 0.329 & 0.079 \\
\hline 4 & ChemPLP@GOLD & 0.579 & 0.697 & 0.818 & 0.776 & 1 & 0.870 & 0.845 & 0.830 & 0.657 & 0.783 & 0.773 & 0.798 & 0.773 & 0.801 & 0.666 & 0.635 & 0.589 & 0.667 & 0.453 & 0.348 & 0.586 & 0.354 \\
\hline 5 & PLP1@DS & 0.568 & 0.781 & 0.880 & 0.657 & 0.870 & 1 & 0.868 & 0.827 & 0.685 & 0.622 & 0.807 & 0.794 & 0.652 & 0.617 & 0.629 & 0.757 & 0.667 & 0.631 & 0.394 & 0.377 & 0.421 & 0.325 \\
\hline 6 & G-Score@SYBYL & 0.558 & 0.707 & 0.820 & 0.692 & 0.845 & 0.868 & 1 & 0.748 & 0.690 & 0.660 & 0.803 & 0.753 & 0.677 & 0.707 & 0.582 & 0.712 & 0.600 & 0.749 & 0.464 & 0.367 & 0.483 & 0.347 \\
\hline 7 & ASP@GOLD & 0.556 & 0.607 & 0.739 & 0.553 & 0.830 & 0.827 & 0.748 & 1 & 0.674 & 0.554 & 0.776 & 0.782 & 0.686 & 0.573 & 0.782 & 0.497 & 0.425 & 0.481 & 0.700 & 0.275 & 0.607 & 0.696 \\
\hline 8 & ASE@MOE & 0.544 & 0.691 & 0.759 & 0.599 & 0.657 & 0.685 & 0.690 & 0.674 & 1 & 0.564 & 0.590 & 0.635 & 0.503 & 0.392 & 0.681 & 0.475 & 0.362 & 0.396 & 0.488 & 0.181 & 0.263 & 0.325 \\
\hline 9 & ChemScore@GOLD & 0.536 & 0.695 & 0.594 & 0.899 & 0.783 & 0.622 & 0.660 & 0.554 & 0.564 & 1 & 0.468 & 0.525 & 0.676 & 0.654 & 0.530 & 0.497 & 0.619 & 0.620 & 0.231 & 0.371 & 0.273 & 0.073 \\
\hline 10 & D-Score@SYBYL & 0.526 & 0.590 & 0.790 & 0.541 & 0.773 & 0.807 & 0.803 & 0.776 & 0.590 & 0.468 & 1 & 0.772 & 0.647 & 0.668 & 0.577 & 0.637 & 0.496 & 0.608 & 0.574 & 0.322 & 0.623 & 0.505 \\
\hline 11 & Alpha-HB@MOE & 0.511 & 0.639 & 0.825 & 0.555 & 0.798 & 0.794 & 0.753 & 0.782 & 0.635 & 0.525 & 0.772 & 1 & 0.595 & 0.634 & 0.617 & 0.580 & 0.441 & 0.546 & 0.549 & 0.241 & 0.532 & 0.452 \\
\hline 12 & LUDI3@DS & 0.488 & 0.573 & 0.556 & 0.774 & 0.773 & 0.652 & 0.677 & 0.686 & 0.503 & 0.676 & 0.647 & 0.595 & 1 & 0.742 & 0.501 & 0.518 & 0.566 & 0.682 & 0.438 & 0.366 & 0.575 & 0.364 \\
\hline 13 & GoldScore@GOLD & 0.483 & 0.461 & 0.607 & 0.701 & 0.801 & 0.617 & 0.707 & 0.573 & 0.392 & 0.654 & 0.668 & 0.634 & 0.742 & 1 & 0.343 & 0.536 & 0.507 & 0.712 & 0.303 & 0.359 & 0.583 & 0.191 \\
\hline 14 & Affinity-dG@MOE & 0.482 & 0.627 & 0.657 & 0.549 & 0.666 & 0.629 & 0.582 & 0.782 & 0.681 & 0.530 & 0.577 & 0.617 & 0.501 & 0.343 & 1 & 0.332 & 0.235 & 0.250 & 0.654 & 0.155 & 0.424 & 0.541 \\
\hline 15 & LigScore2@DS & 0.456 & 0.636 & 0.720 & 0.523 & 0.635 & 0.757 & 0.712 & 0.497 & 0.475 & 0.497 & 0.637 & 0.580 & 0.518 & 0.536 & 0.332 & 1 & 0.707 & 0.603 & 0.233 & 0.449 & 0.218 & 0.091 \\
\hline 16 & GlideScore-SP & 0.452 & 0.585 & 0.537 & 0.614 & 0.589 & 0.667 & 0.600 & 0.425 & 0.362 & 0.619 & 0.496 & 0.441 & 0.566 & 0.507 & 0.235 & 0.707 & 1 & 0.659 & 0.018 & 0.673 & 0.180 & -0.058 \\
\hline 17 & Jain@DS & 0.408 & 0.440 & 0.490 & 0.668 & 0.667 & 0.631 & 0.749 & 0.481 & 0.396 & 0.620 & 0.608 & 0.546 & 0.682 & 0.712 & 0.250 & 0.603 & 0.659 & 1 & 0.222 & 0.517 & 0.523 & 0.156 \\
\hline 18 & PMF@DS & 0.364 & 0.338 & 0.461 & 0.272 & 0.453 & 0.394 & 0.464 & 0.700 & 0.488 & 0.231 & 0.574 & 0.549 & 0.438 & 0.303 & 0.654 & 0.233 & 0.018 & 0.222 & 1 & 0.003 & 0.561 & 0.883 \\
\hline 19 & GlideScore-XP & 0.278 & 0.310 & 0.270 & 0.395 & 0.348 & 0.377 & 0.367 & 0.275 & 0.181 & 0.371 & 0.322 & 0.241 & 0.366 & 0.359 & 0.155 & 0.449 & 0.673 & 0.517 & 0.003 & 1 & 0.214 & -0.001 \\
\hline 20 & London-dG@MOE & 0.241 & 0.094 & 0.353 & 0.329 & 0.586 & 0.421 & 0.483 & 0.607 & 0.263 & 0.273 & 0.623 & 0.532 & 0.575 & 0.583 & 0.424 & 0.218 & 0.180 & 0.523 & 0.561 & 0.214 & 1 & 0.610 \\
\hline 21 & PMF@SYBYL & 0.221 & 0.108 & 0.287 & 0.079 & 0.354 & 0.325 & 0.347 & 0.696 & 0.325 & 0.073 & 0.505 & 0.452 & 0.364 & 0.191 & 0.541 & 0.091 & -0.058 & 0.156 & 0.883 & -0.001 & 0.610 & 1 \\
\hline
\end{tabular}

$a$ : Correlation coefficient between the experimental binding constants and the predictions given by this scoring function.

$b$ : Inter-correlation coefficient between the predictions given by the two relevant scoring functions, which are colored in three tiers $: R_{\mathrm{AB}} \geq 0.6$ (red); $R_{\mathrm{AB}}=0.3 \sim 0.6$

(orange); $R_{\mathrm{AB}}<0.3$ (green). 
Table S22. Comparing all scoring functions in pair by their correlation coefficients with our method $(95 \% \text { confidence and power }=0.80)^{\text {a }}$

\begin{tabular}{|c|c|c|c|c|c|c|c|c|c|c|c|c|c|c|c|c|c|c|c|c|c|c|c|}
\hline \multirow{2}{*}{ Rank } & \multirow{2}{*}{ Scoring function } & \multirow{2}{*}{$R$} & \multicolumn{21}{|c|}{ Whether the two correlations are significantly different at the $95 \%$ confidence level } \\
\hline & & & $\# 1$ & \#2 & \#3 & \#4 & $\# 5$ & \#6 & \#7 & \#8 & \#9 & $\# 10$ & \#11 & $\# 12$ & $\# 13$ & \#14 & $\# 15$ & $\# 16$ & \#17 & $\# 18$ & $\# 19$ & $\# 20$ & \#21 \\
\hline 1 & X-ScoreHM & 0.614 & & No & No & No & No & No & No & No & Yes & Yes & Yes & Yes & Yes & Yes & Yes & Yes & Yes & Yes & Yes & Yes & Yes \\
\hline 2 & $\Delta \mathrm{SAS}$ & 0.606 & No & & No & No & No & No & No & No & No & Yes & Yes & Yes & Yes & Yes & Yes & Yes & Yes & Yes & Yes & Yes & Yes \\
\hline 3 & ChemScore@SYBYL & 0.592 & No & No & & No & No & No & No & No & Yes & No & No & Yes & Yes & Yes & Yes & Yes & Yes & Yes & Yes & Yes & Yes \\
\hline 4 & ChemPLP@GOLD & 0.579 & No & No & No & & No & No & No & No & No & No & Yes & Yes & Yes & Yes & Yes & Yes & Yes & Yes & Yes & Yes & Yes \\
\hline 5 & PLP1@DS & 0.568 & No & No & No & No & & No & No & No & No & No & No & No & Yes & Yes & Yes & Yes & Yes & Yes & Yes & Yes & Yes \\
\hline 6 & G-Score@SYBYL & 0.558 & No & No & No & No & No & & No & No & No & No & No & No & No & No & Yes & Yes & Yes & Yes & Yes & Yes & Yes \\
\hline 7 & ASP@GOLD & 0.556 & No & No & No & No & No & No & & No & No & No & No & No & No & Yes & Yes & Yes & Yes & Yes & Yes & Yes & Yes \\
\hline 8 & ASE@MOE & 0.544 & No & No & No & No & No & No & No & & No & No & No & No & No & No & No & No & Yes & Yes & Yes & Yes & Yes \\
\hline 9 & ChemScore@GOLD & 0.536 & Yes & No & Yes & No & No & No & No & No & & No & No & No & No & No & No & No & Yes & Yes & Yes & Yes & Yes \\
\hline 10 & D-Score@SYBYL & 0.526 & Yes & Yes & No & No & No & No & No & No & No & & No & No & No & No & No & No & Yes & Yes & Yes & Yes & Yes \\
\hline 11 & Alpha-HB @MOE & 0.511 & Yes & Yes & No & Yes & No & No & No & No & No & No & 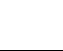 & No & No & No & No & No & Yes & Yes & Yes & Yes & Yes \\
\hline 12 & LUDI3@DS & 0.488 & Yes & Yes & Yes & Yes & No & No & No & No & No & No & No & & No & No & No & No & No & Yes & Yes & Yes & Yes \\
\hline 13 & GoldScore@GOLD & 0.483 & Yes & Yes & Yes & Yes & Yes & No & No & No & No & No & No & No & . & No & No & No & No & No & Yes & Yes & Yes \\
\hline 14 & Affinity-dG@MOE & 0.482 & Yes & Yes & Yes & Yes & Yes & No & Yes & No & No & No & No & No & No & & No & No & No & Yes & Yes & Yes & Yes \\
\hline 15 & LigScore2@DS & 0.456 & Yes & Yes & Yes & Yes & Yes & Yes & Yes & No & No & No & No & No & No & No & 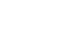 & No & No & No & Yes & Yes & Yes \\
\hline 16 & GlideScore-SP & 0.452 & Yes & Yes & Yes & Yes & Yes & Yes & Yes & No & No & No & No & No & No & No & No & & No & No & Yes & Yes & Yes \\
\hline 17 & Jain@DS & 0.408 & Yes & Yes & Yes & Yes & Yes & Yes & Yes & Yes & Yes & Yes & Yes & No & No & No & No & No & & No & Yes & Yes & Yes \\
\hline 18 & PMF@DS & 0.364 & Yes & Yes & Yes & Yes & Yes & Yes & Yes & Yes & Yes & Yes & Yes & Yes & No & Yes & No & No & No & & No & Yes & Yes \\
\hline 19 & GlideScore-XP & 0.278 & Yes & Yes & Yes & Yes & Yes & Yes & Yes & Yes & Yes & Yes & Yes & Yes & Yes & Yes & Yes & Yes & Yes & No & & No & No \\
\hline 20 & London-dG@MOE & 0.241 & Yes & Yes & Yes & Yes & Yes & Yes & Yes & Yes & Yes & Yes & Yes & Yes & Yes & Yes & Yes & Yes & Yes & Yes & No & & No \\
\hline 21 & PMF@SYBYL & 0.221 & Yes & Yes & Yes & Yes & Yes & Yes & Yes & Yes & Yes & Yes & Yes & Yes & Yes & Yes & Yes & Yes & Yes & Yes & No & No & \\
\hline
\end{tabular}

$a$ : It is labeled as "Yes" if the Dunn \& Clark test statistic computed for the two relevant scoring functions is larger than the critical value of 1.64; otherwise it is labeled as "No". 
Table S23. Comparing all scoring functions in pair by their correlation coefficients with our method $(90 \%$ confidence and power $=0.80)$

\begin{tabular}{|c|c|c|c|c|c|c|c|c|c|c|c|c|c|c|c|c|c|c|c|c|c|c|c|}
\hline \multirow{2}{*}{ Rank } & \multirow{2}{*}{ Scoring function } & \multirow{2}{*}{$R$} & \multicolumn{21}{|c|}{ Whether the two correlations are significantly different at the $90 \%$ confidence level } \\
\hline & & & $\# 1$ & $\# 2$ & $\# 3$ & $\# 4$ & $\# 5$ & $\# 6$ & $\# 7$ & $\# 8$ & $\# 9$ & $\# 10$ & $\# 11$ & $\# 12$ & $\# 13$ & $\# 14$ & $\# 15$ & $\# 16$ & $\# 17$ & $\# 18$ & $\# 19$ & $\# 20$ & $\# 21$ \\
\hline 1 & X-ScoreHM & 0.614 & & No & No & No & No & Yes & No & Yes & Yes & Yes & Yes & Yes & Yes & Yes & Yes & Yes & Yes & Yes & Yes & Yes & Yes \\
\hline 2 & $\triangle \mathrm{SAS}$ & 0.606 & No & & No & No & Yes & Yes & No & Yes & Yes & Yes & Yes & Yes & Yes & Yes & Yes & Yes & Yes & Yes & Yes & Yes & Yes \\
\hline 3 & ChemScore@SYBYL & 0.592 & No & No & & No & No & No & No & No & Yes & No & Yes & Yes & Yes & Yes & Yes & Yes & Yes & Yes & Yes & Yes & Yes \\
\hline 4 & ChemPLP@GOLD & 0.579 & No & No & No & & No & No & No & No & No & Yes & Yes & Yes & Yes & Yes & Yes & Yes & Yes & Yes & Yes & Yes & Yes \\
\hline 5 & PLP1@DS & 0.568 & No & Yes & No & No & & No & No & No & No & No & Yes & Yes & Yes & Yes & Yes & Yes & Yes & Yes & Yes & Yes & Yes \\
\hline 6 & G-Score@SYBYL & 0.558 & Yes & Yes & No & No & No & & No & No & No & No & No & Yes & Yes & Yes & Yes & Yes & Yes & Yes & Yes & Yes & Yes \\
\hline 7 & ASP@GOLD & 0.556 & No & No & No & No & No & No & & No & No & No & No & Yes & Yes & Yes & Yes & Yes & Yes & Yes & Yes & Yes & Yes \\
\hline 8 & ASE@MOE & 0.544 & Yes & Yes & No & No & No & No & No & & No & No & No & No & No & Yes & Yes & Yes & Yes & Yes & Yes & Yes & Yes \\
\hline 9 & ChemScore@GOLD & 0.536 & Yes & Yes & Yes & No & No & No & No & No & & No & No & No & No & No & Yes & Yes & Yes & Yes & Yes & Yes & Yes \\
\hline 10 & D-Score@SYBYL & 0.526 & Yes & Yes & No & Yes & No & No & No & No & No & & No & No & No & No & Yes & No & Yes & Yes & Yes & Yes & Yes \\
\hline 11 & Alpha-HB@MOE & 0.511 & Yes & Yes & Yes & Yes & Yes & No & No & No & No & No & & No & No & No & No & No & Yes & Yes & Yes & Yes & Yes \\
\hline 12 & LUDI3@DS & 0.488 & Yes & Yes & Yes & Yes & Yes & Yes & Yes & No & No & No & No & & No & No & No & No & Yes & Yes & Yes & Yes & Yes \\
\hline 13 & GoldScore@GOLD & 0.483 & Yes & Yes & Yes & Yes & Yes & Yes & Yes & No & No & No & No & No & . & No & No & No & Yes & Yes & Yes & Yes & Yes \\
\hline 14 & Affinity-dG@MOE & 0.482 & Yes & Yes & Yes & Yes & Yes & Yes & Yes & Yes & No & No & No & No & No & & No & No & No & Yes & Yes & Yes & Yes \\
\hline 15 & LigScore2@DS & 0.456 & Yes & Yes & Yes & Yes & Yes & Yes & Yes & Yes & Yes & Yes & No & No & No & No & & No & No & No & Yes & Yes & Yes \\
\hline 16 & GlideScore-SP & 0.452 & Yes & Yes & Yes & Yes & Yes & Yes & Yes & Yes & Yes & No & No & No & No & No & No & & No & No & Yes & Yes & Yes \\
\hline 17 & Jain@DS & 0.408 & Yes & Yes & Yes & Yes & Yes & Yes & Yes & Yes & Yes & Yes & Yes & Yes & Yes & No & No & No & & No & Yes & Yes & Yes \\
\hline 18 & PMF@DS & 0.364 & Yes & Yes & Yes & Yes & Yes & Yes & Yes & Yes & Yes & Yes & Yes & Yes & Yes & Yes & No & No & No & & No & Yes & Yes \\
\hline 19 & GlideScore-XP & 0.278 & Yes & Yes & Yes & Yes & Yes & Yes & Yes & Yes & Yes & Yes & Yes & Yes & Yes & Yes & Yes & Yes & Yes & No & & No & No \\
\hline 20 & London-dG@MOE & 0.241 & Yes & Yes & Yes & Yes & Yes & Yes & Yes & Yes & Yes & Yes & Yes & Yes & Yes & Yes & Yes & Yes & Yes & Yes & No & & No \\
\hline 21 & PMF@SYBYL & 0.221 & Yes & Yes & Yes & Yes & Yes & Yes & Yes & Yes & Yes & Yes & Yes & Yes & Yes & Yes & Yes & Yes & Yes & Yes & No & No & \\
\hline
\end{tabular}

$a$ : It is labeled as "Yes" if the Dunn \& Clark test statistic computed for the two relevant scoring functions is larger than the critical value of 1.28; otherwise it is labeled as "No". 


\section{PART 7. SOURCE CODES USED IN NUMERICAL SIMULATION}

All of the following programs should be run within the $R$ software. One can run them directly in the Console window in the GUI. Or, one can save them as files, load them via File > Open script, and then run them via Edit > Run all. The results will be output in a few minutes.

\section{Codes for generating the desired mother sets}

Three separate functions (named "data") were used to generate the mother sets in three different forms and diverse configurations. The input is a vector specifying the three desired correlation coefficients, i.e. rhoA, rhoB and rho AB. The output is a matrix with 3 columns and 100,000 rows, where each row represents a hypothetical sample. The correlations between these rows are rhoA, rhoB and rho $\mathrm{AB}$, respectively.

\subsection{Generate mother sets in normal distribution}

\#\#\# Normal Distribution Mother Set \#\#\#

library(MASS)

data $<-$ function $(\mathrm{COR})\{$

\# COR is the correlations vector: rhoA, rhoB, and rho $A B$

\# correlation matrix

$\# 1 \times y$

$\# x 1$ y

$\#$ y z 1

\# define correlation matrix

$x<-\operatorname{COR}[1] ; y<-\operatorname{COR}[2] ; z<-\operatorname{COR}[3]$

rho $<-\operatorname{matrix}(c(1, x, y, x, 1, z, y, z, 1), 3,3)$

cova $<-$ rho $* 2$

\# Random Number Generation

set.seed(1)

\# $\mathrm{X} \sim \mathrm{N}(7,2)$

data <- mvrnorm(100000, c $(7,7,7)$, cova, empirical $=\mathrm{T})$

data $<-$ round(data, 2)

return(data)

\}

\subsection{Generate mother sets in uniform distribution}

\#\#\# Uniform Distribution Mother Set \#\#\# 


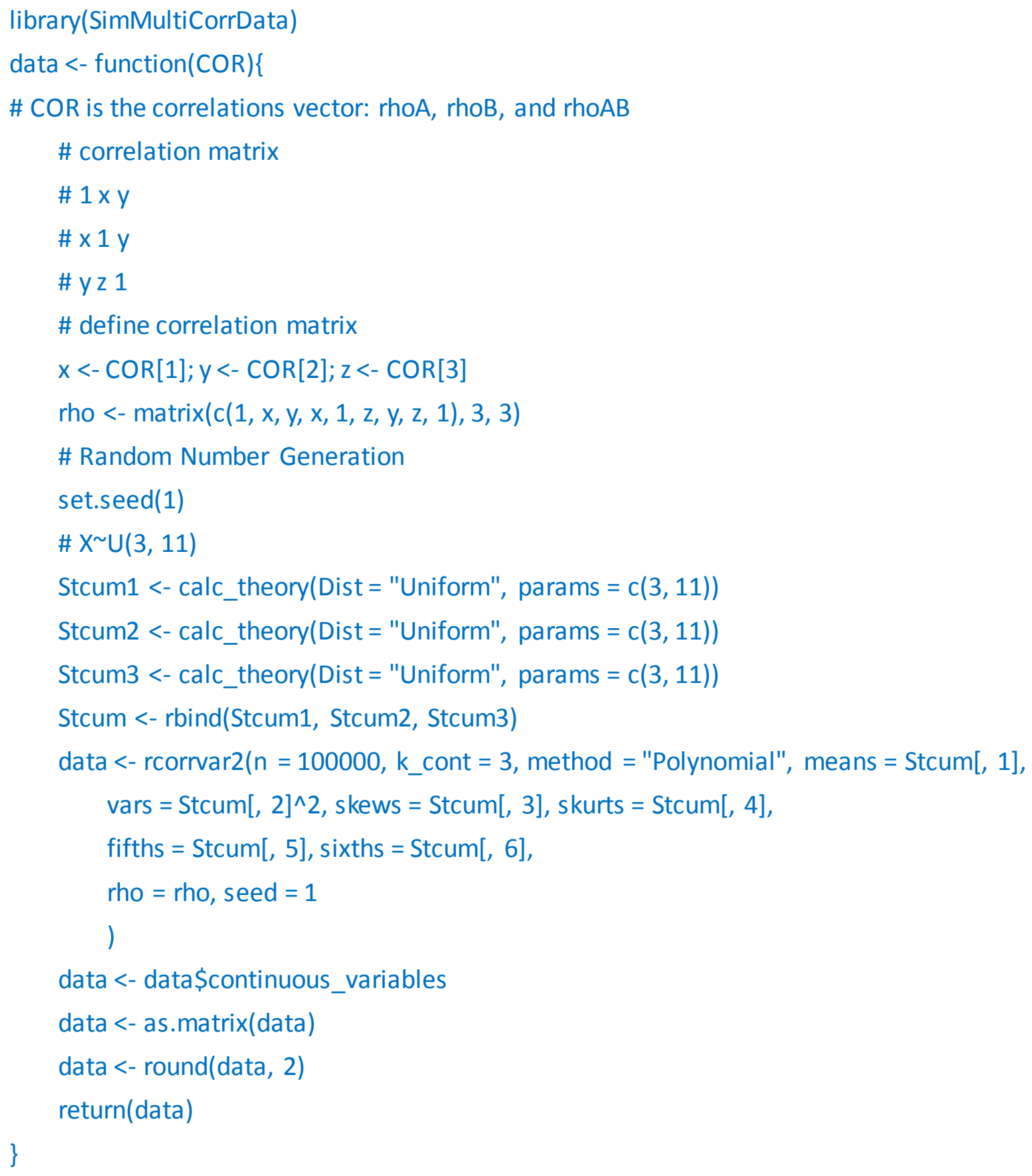

\subsection{Generating mother sets in exponential distribution}

\#\#\# Exponential Distribution Mother Set \#\#\#

library(SimMultiCorrData)

data $<-$ function $(C O R)\{$

\# COR is the correlations vector: rhoA, rhoB, and rhoAB

\# correlation matrix

\# 1 x y

\# $x 1$ y

\# y $z 1$ 


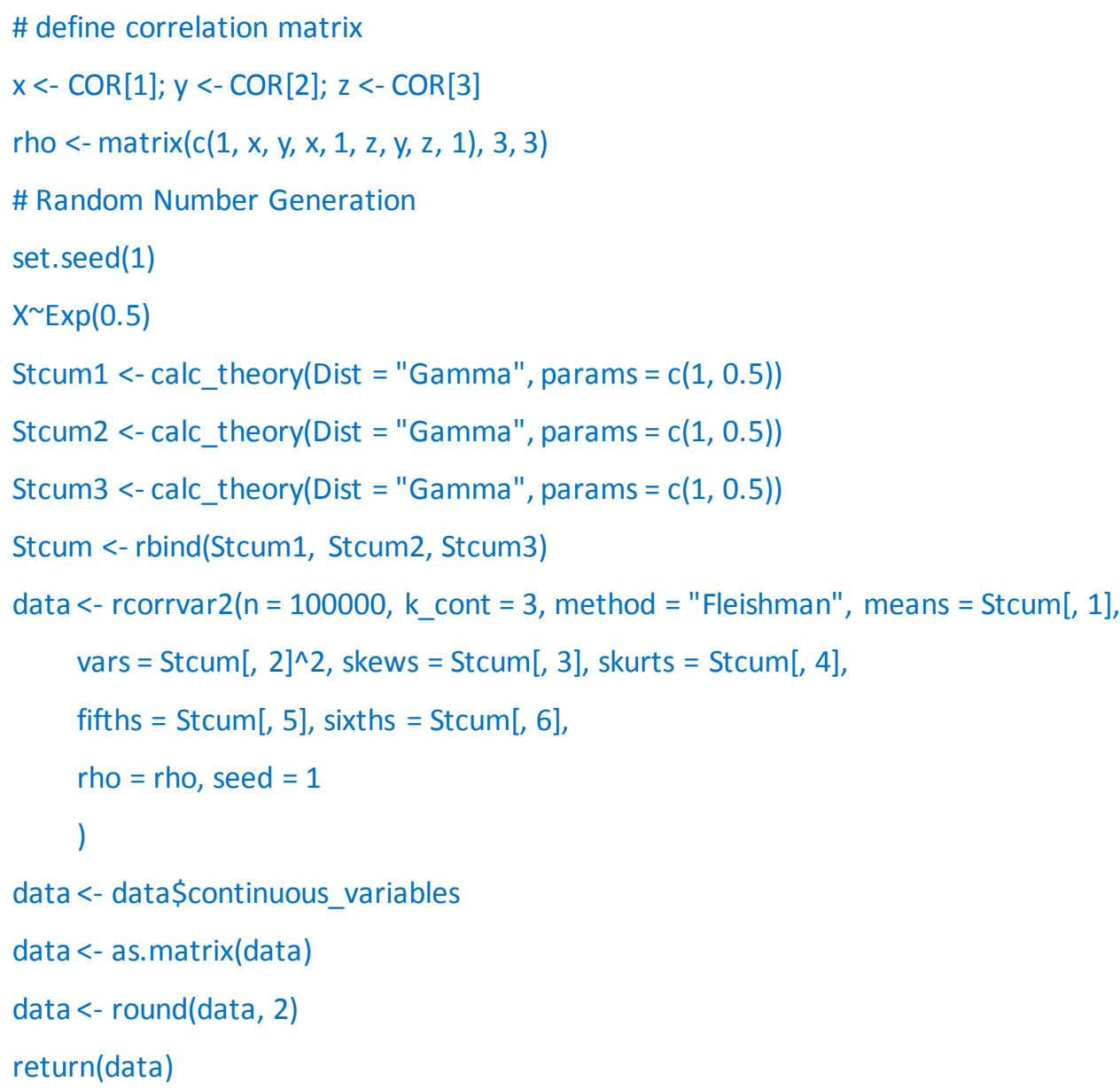

\section{Codes for calculating test statistic}

Two functions (named "stati") were used to calculate the Carlson's test statistic and Dunn \& Clark's test statistic, respectively. The input includes a matrix of mother set data and sample size $N$ specified by the user. The output is a vector with 10,000 elements, which are the computed statistics from 10,000 trials.

\subsection{Calculate Carlson's test statistic}

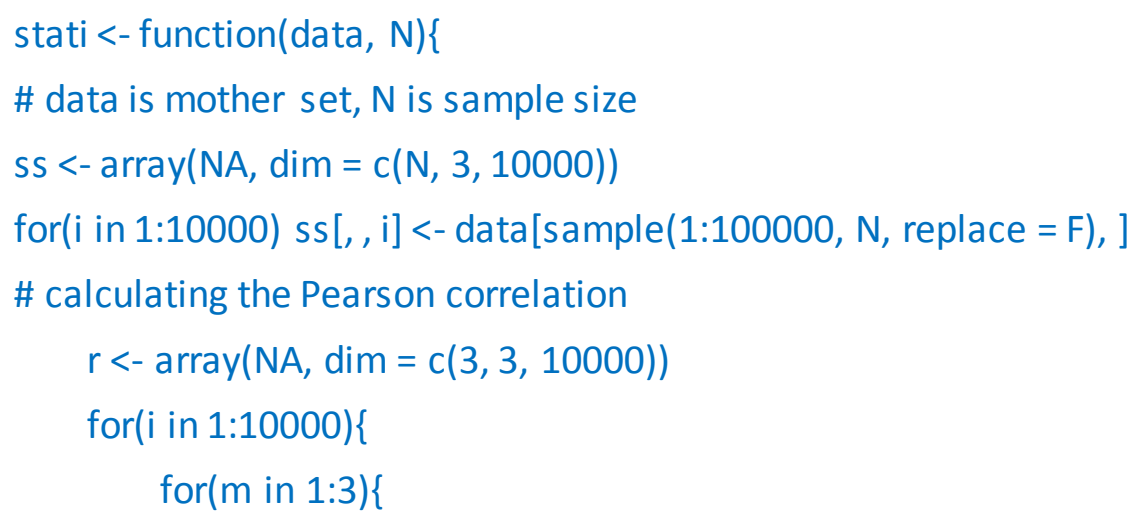




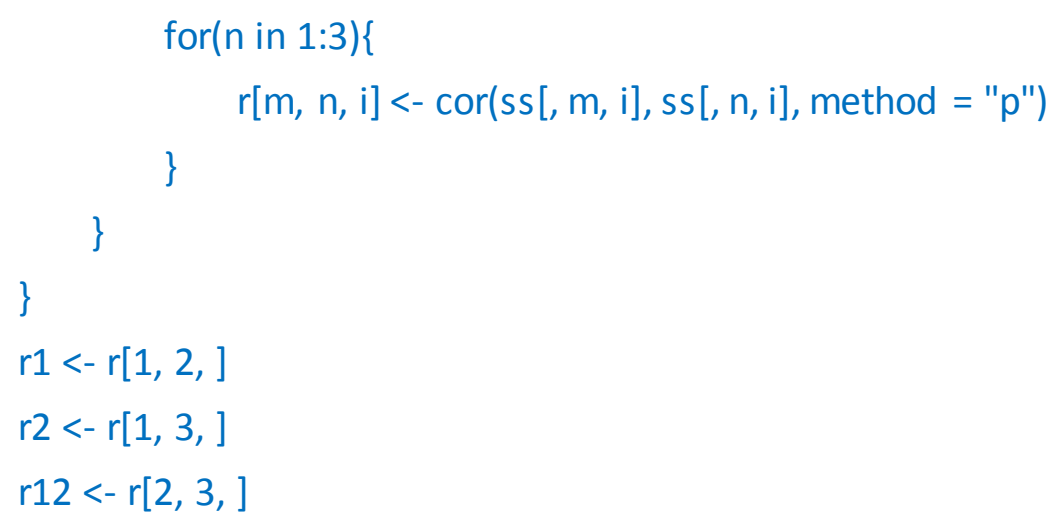

\subsection{Calculate Dunn \& Clark's test statistic}

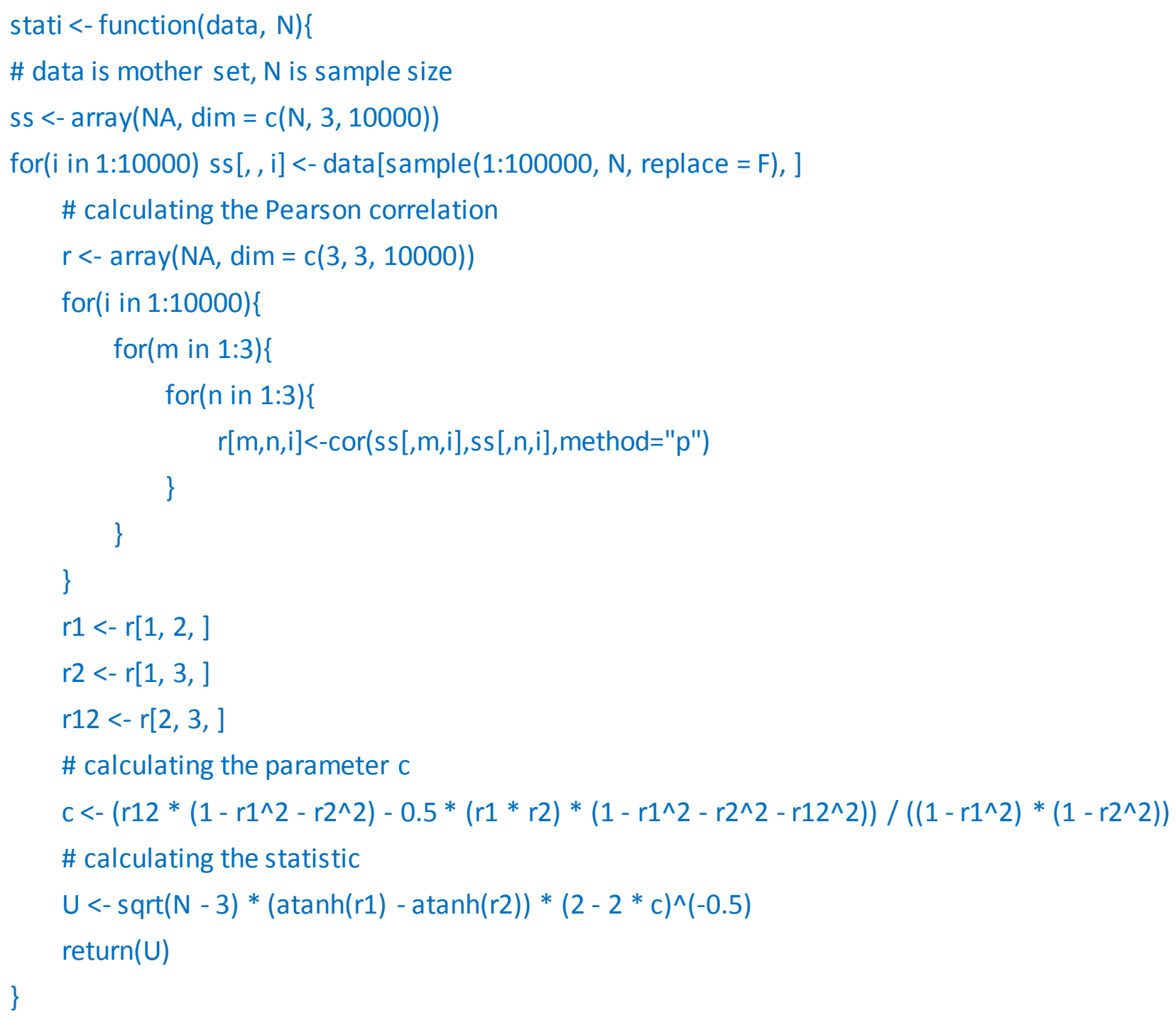




\section{Codes for calculating type I error}

In order to analyze type I error, we calculated the test statistics obtained based on mother sets at diverse configurations. All 17 configurations considered in our study are summarized in Table 2 in the main text of our manuscript. The following program needs to call functions "stati" and "data". Thus, one needs to run those two functions in the $R$ software in prior. Its output includes four files containing the results obtained at sample size of 50,100, 200, and 500. Each file contains a matrix with 17 columns and 10,000 rows. Each column contains the test statistic computed from 10,000 trials based on the given mother set with a specific configuration.

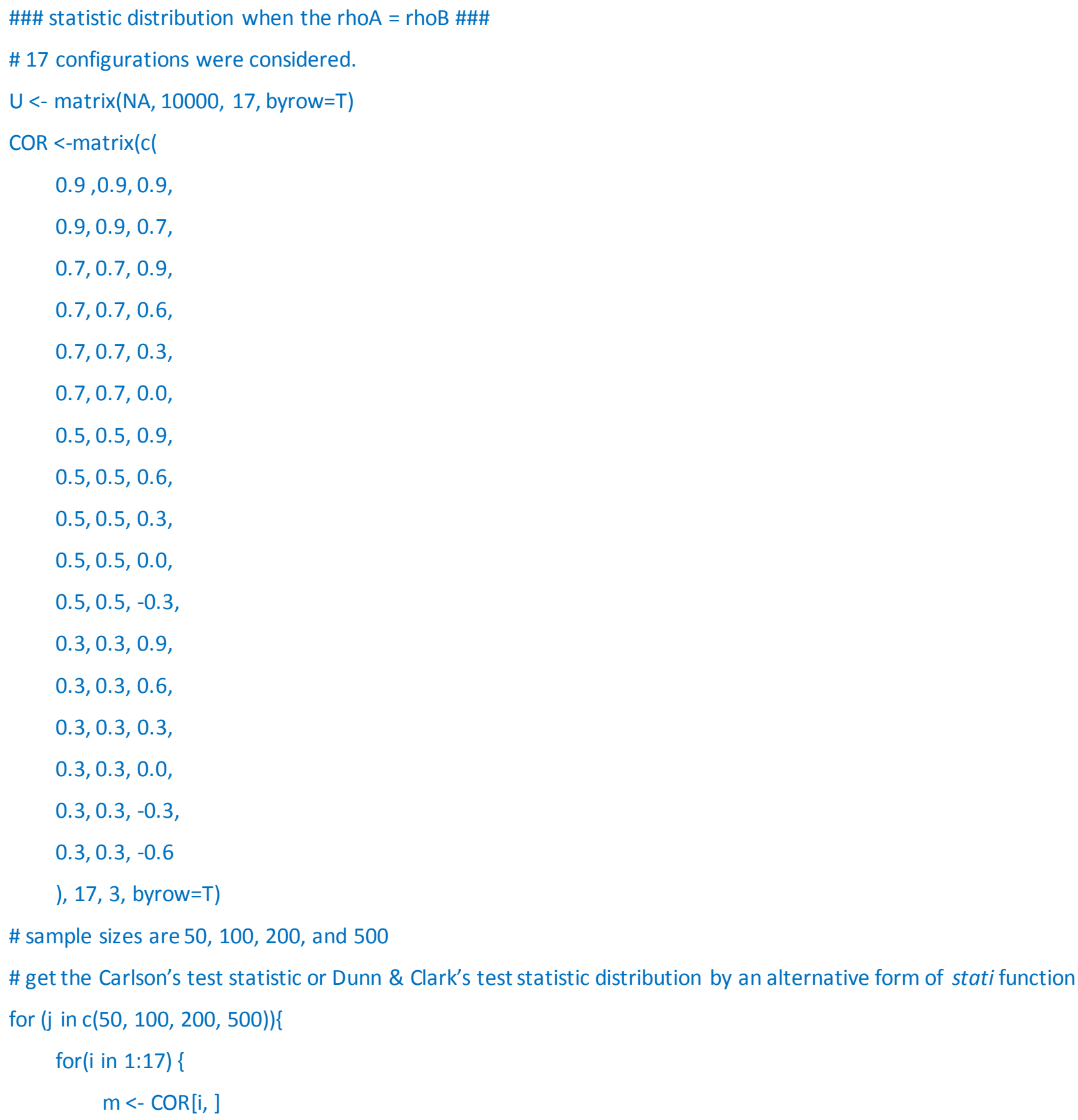




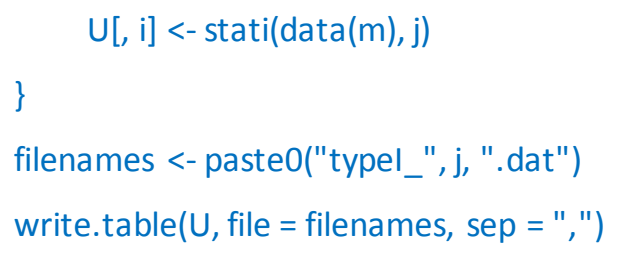

\section{Codes for calculating type II error}

In order to analyze type II error, we calculated the test statistics obtained based on mother sets at diverse configurations. All 18 configurations considered in our study are summarized in Table 3 in the main text of our manuscript. The following program needs to call functions "stati" (for Dunn $\&$ Clark's test statistic) and "data". One needs to run those two functions in the $R$ software in prior. The output includes four files in different formats. The first file contains a matrix of 4 columns and 10,000 rows, which records the test statistic obtained on mother set ID 5-1 to 5-4. The second file contains a matrix of 5 columns and 10,000 rows, which records the test statistic obtained on mother set ID 6-1 to 6-5. The third file contains a matrix of 4 columns and 10,000 rows, which records the test statistic obtained on mother set ID 7-1 to 7-4. The fourth file contains a matrix of 5 columns and 10,000 rows, which records the test statistic obtained on mother set ID 8-1 to 8-5.

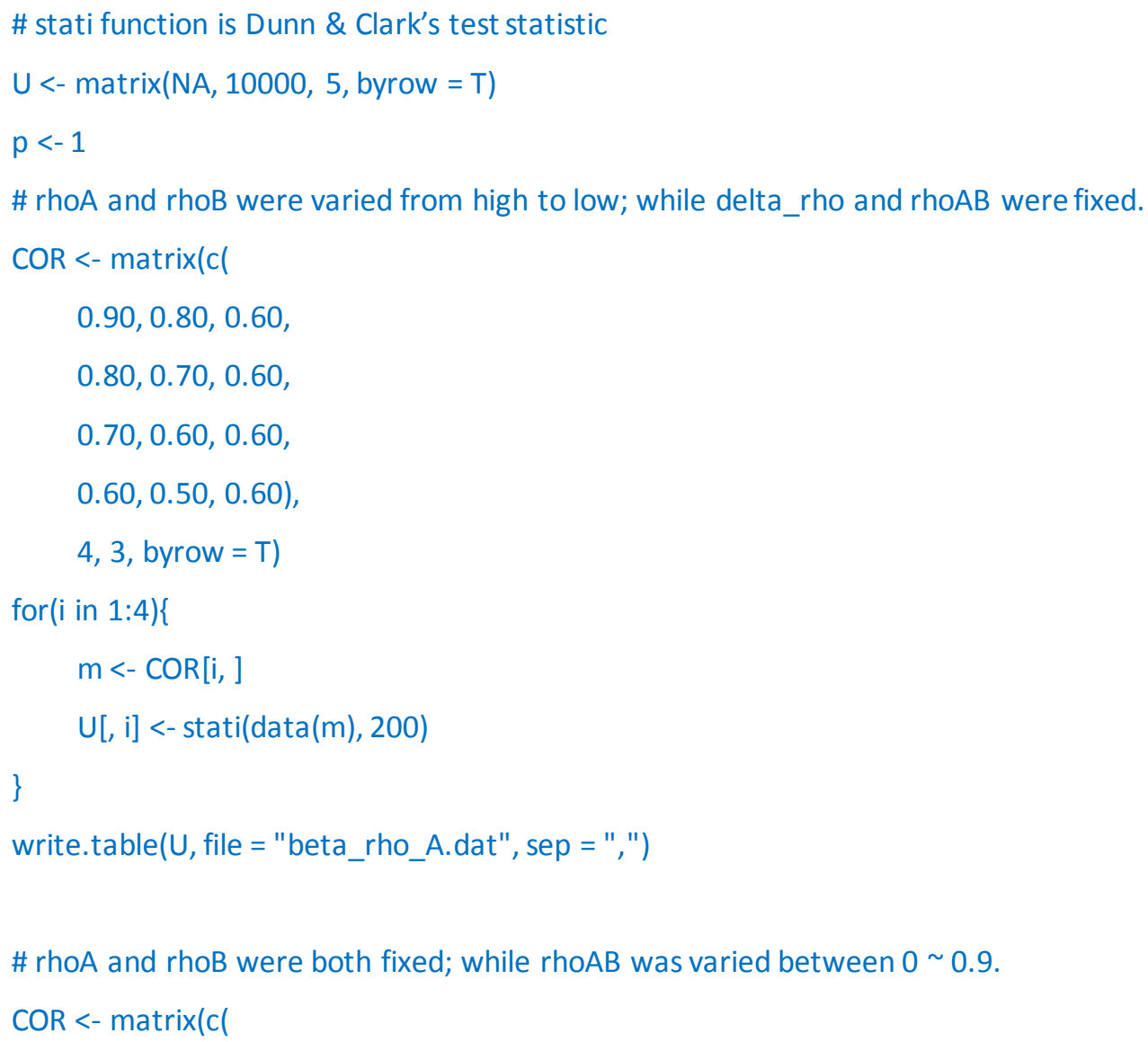




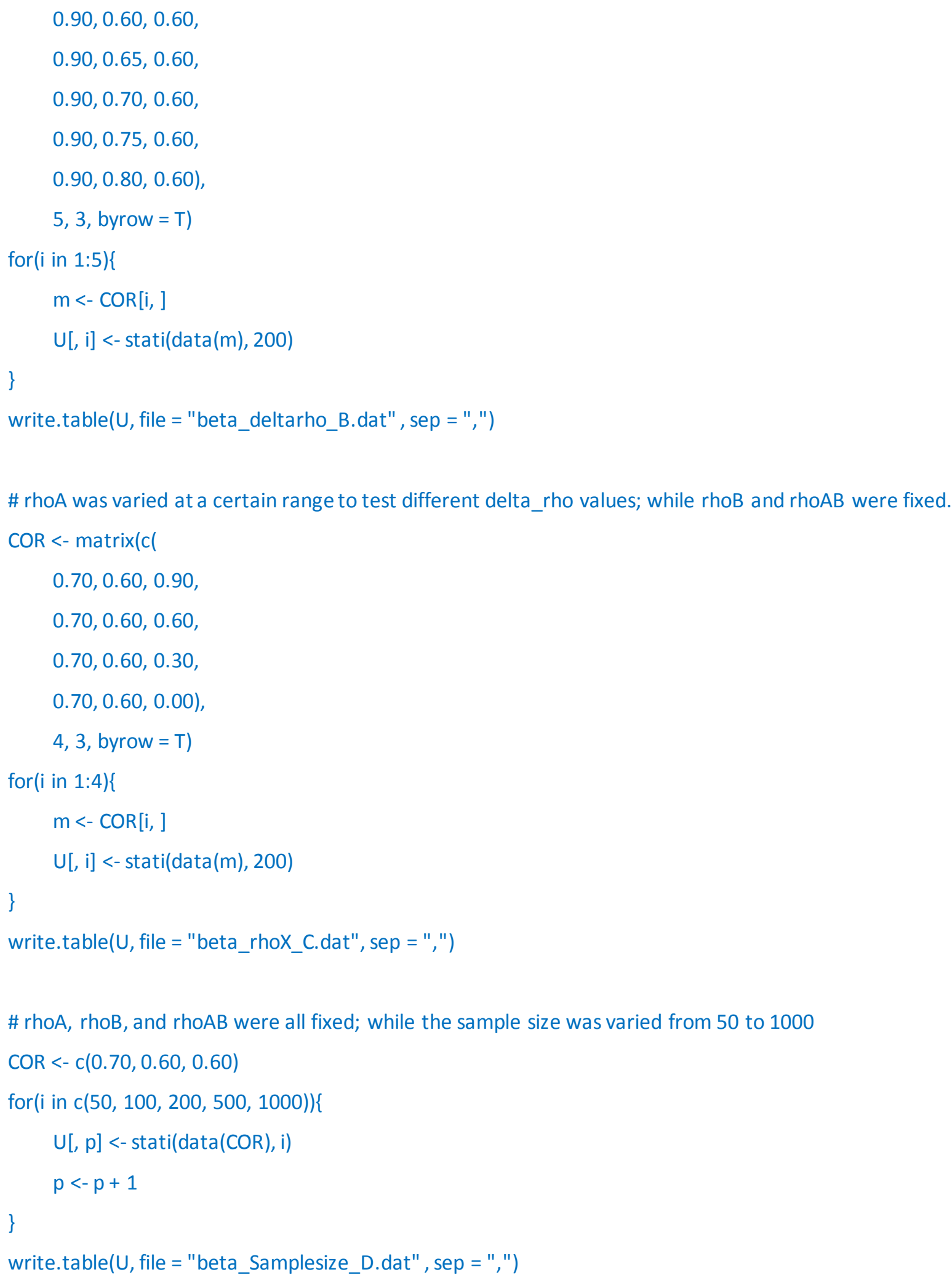

\section{Codes for performing the normality test}

In order to test the normality of each distribution of the test statistic, we applied the Anderson-Darling test enabled in the $R$ software. The input is a matrix containing $M$ columns and 
10,000 rows. Here, $M$ is the total number of mother sets in the current evaluation. For example, $M=$ 17 when the mother sets in normal distribution were under consideration (see Table 2 in the main text). Each row records the test statistic derived from a single trial. The output of this program records the Anderson-Darling test result (p-value), mean, and standard deviation of every column.

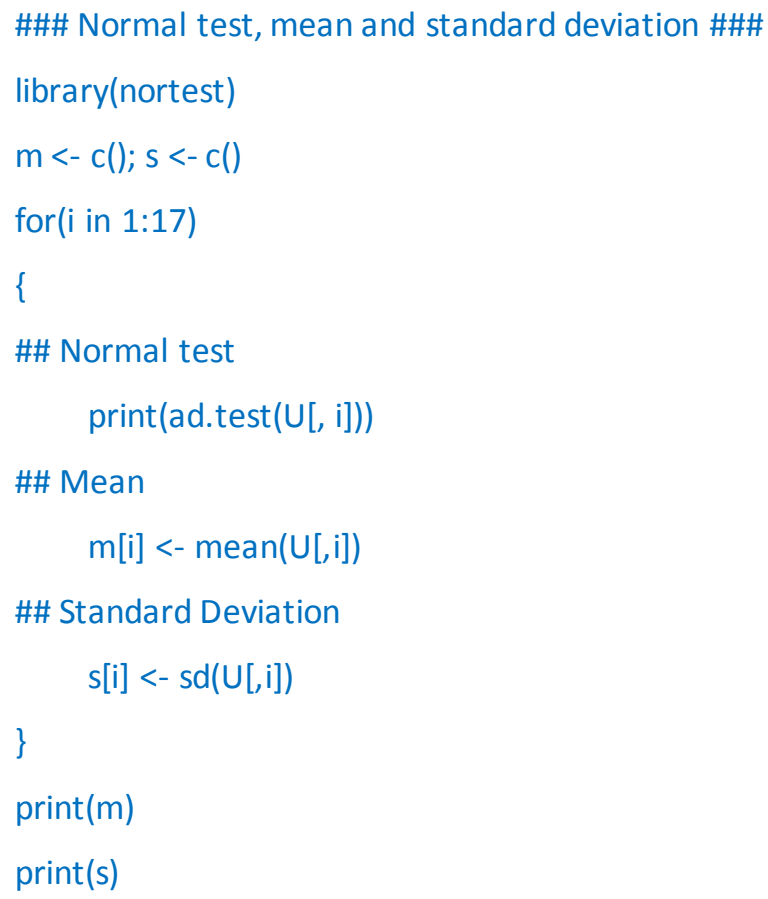

